Document downloaded from:

http://hdl.handle.net/10251/155861

This paper must be cited as:

Defant, A.; Mastylo, M.; Sánchez Pérez, EA.; Steinwart, I. (2019). Translation invariant maps on function spaces over locally compact groups. Journal of Mathematical Analysis and Applications. 470(2):795-820. https://doi.org/10.1016/j.jmaa.2018.10.033

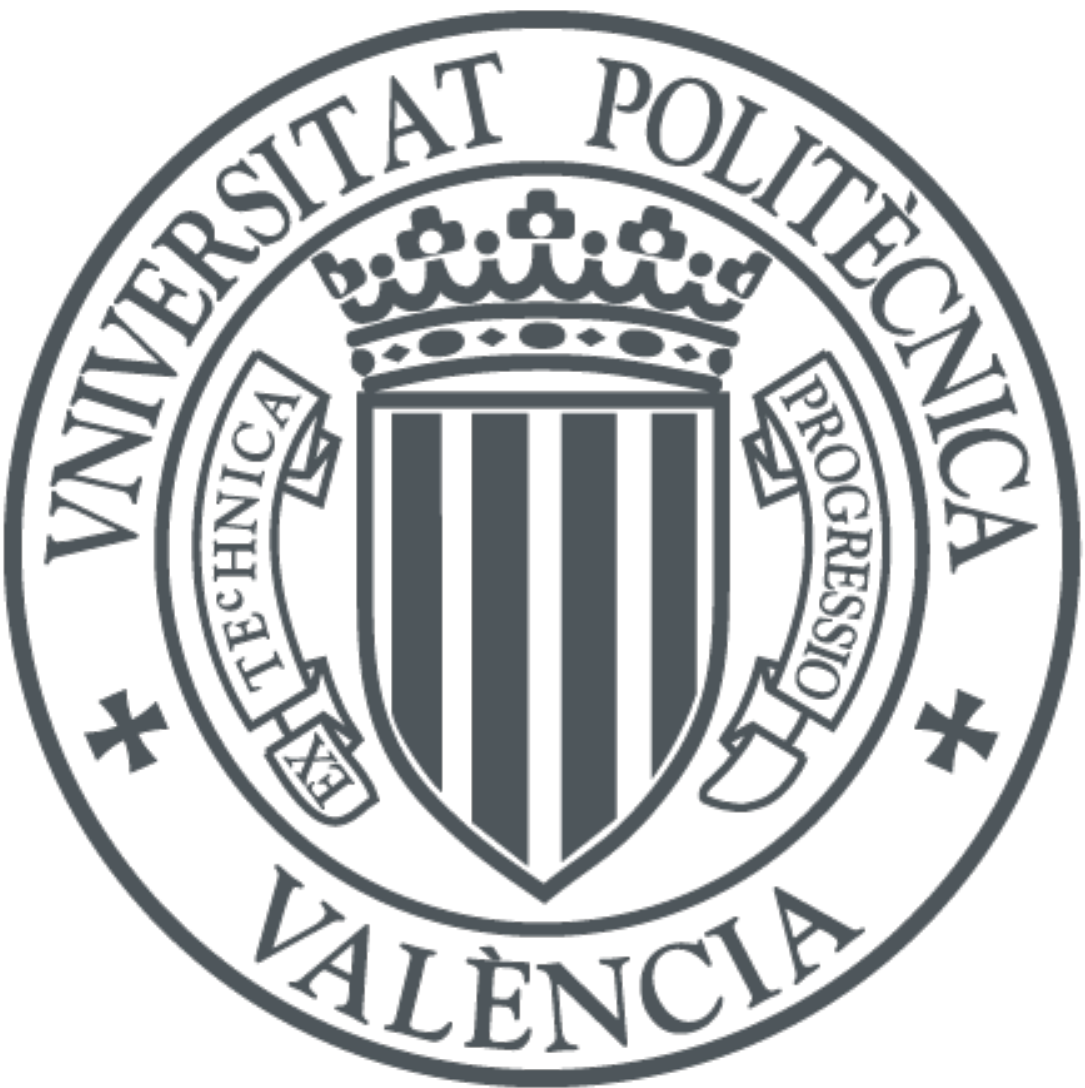

The final publication is available at

https://doi.org/10.1016/j.jmaa.2018.10.033

Copyright Elsevier

Additional Information 


\title{
TRANSLATION INVARIANT MAPS ON FUNCTION SPACES OVER LOCALLY COMPACT GROUPS
}

\author{
A. DEFANT, M. MASTYŁO, E. A. SÁNCHEZ-PÉREZ, AND I. STEINWART
}

\begin{abstract}
We prove that under adequate geometric requirements, translation invariant mappings between vector-valued quasi-Banach function spaces on a locally compact group $G$ have a bounded extension between KötheBochner spaces $L_{r}(G, E)$. The class of mappings for which our results apply includes polynomials and multilinear operators. We develop an abstract approach based on some new tools as abstract convolution and matching among Banach function lattices, and also on some classical techniques as MaureyRosenthal factorization of operators. As a by-product we show when Haar measures which appear in certain factorization theorems for nonlinear mappings are in fact Pietsch measures. We also give applications to operators between Köthe-Bochner spaces.
\end{abstract}

\section{Introduction}

Our aim is to establish an abstract approach to study $L_{r}$-boundedness of (non linear in general) translation invariant mappings between scalar- and vector-valued quasi Banach spaces. We point out that the class of translation invariant mappings is of interest in various areas of harmonic analysis and functional analysis. The theory of translation invariant operators is a sort of interplay between operator theory and function theory on locally compact groups applied with the Haar measure. As an example, we mention that a combination of well-known theorems due to Nikishin and Stein with the theory of locally compact groups found deep applications in the factorization theory of operators and in the theory of orthogonal series.

Relevant results on this topic were proved in the seventies and eighties using different techniques. Among others, we mention here the works of Colzani [3] Cowling and Fournier [5, 6], Herz and Riviéra [18, 19], Larsen [23], Oberlin [28] and Shternberg [33]. Special attention was paid to quasi-Banach spaces, and interesting

2010 Mathematics Subject Classification. 47B38, 46E30.

Key words and phrases. Banach function lattices, translation invariant operators, Haar measure.

The second named author was supported by National Science Centre, Poland, project, no. 2015/17/B/ST1/00064. The third named author was supported by Ministerio de Economía, Industria y Competitividad (Spain) and FEDER (project MTM2016-77054-C2-1-P2). 
representation results for translation invariant operators were obtained for this case. We refer to the papers by Oberlin ([26, 27]), Colzani and Sjögren [4], and Sjögren [34], where translation invariant operators on $L_{p}$-spaces $(0<p<1)$ and related spaces including Lorentz spaces are investigated. All these papers present interesting results with useful applications, and use techniques coming from classical analysis.

The aim of the present paper is to use some modern techniques to address this classical topic, presenting a unified approach for understanding it in a general framework and using it to generalize the main results to broad classes of operators and quasi-Banach lattices of measurable functions. Thus, the analysis of the following question summarizes the main objective of our study: Let $G$ be a locally compact group with left Haar measure $\lambda$. Consider a translation invariant $m$-homogeneous map $\Phi$ from a Banach function lattice $X(\lambda)$ to a Banach function lattice $Y(\lambda)$. Under which requirements can we state that $\Phi$ extends to a translation invariant m-homogeneous map from $L_{r}(\lambda)$ to $L_{r / m}(\lambda)$ ?

We emphasize that our technique differs from the ones used in the above mentioned papers. The main idea is as follows. Given a bounded mapping between vector-valued quasi-Banach spaces we intend to prove boundeness between a new scale of spaces. This is deduced under the hypothesis that the operator satisfies certain invariance properties and vector-valued geometrical inequalities.

Our method is inspired by the classical book by García Cuerva and Rubio de Francia [15], and uses factorization arguments having their roots in the fundamental work of Maurey and Rosenthal.

\section{Preliminaries}

We will use standard notation from Banach space theory. If $(E,\|\cdot\|)$ is a quasiBanach space, we write $S_{E}=\left\{x \in X:\|x\|_{E}=1\right\}$ for its unit sphere. Throughout the paper let $(\Omega, \Sigma, \mu)$ denote a complete and $\sigma$-finite measure space. As usual $L^{0}(\Omega, \Sigma, \mu)\left(L^{0}(\mu)\right.$ or $L^{0}(\Omega)$ for short $)$ denotes the space of all equivalence classes of real-valued measurable functions on $\Omega$. Given $0<m<\infty$, a mapping $\Phi: E \rightarrow F$ between quasi-Banach spaces is said to be an $m$-homogeneous operator whenever $\Phi(\lambda x)=\lambda^{m} \Phi(x)$ for every $\lambda \geq 0$ and all $x \in X$. Such operators are said to be bounded if there is $C>0$ such that $\|\Phi(x)\| \leq C\|x\|$ for all $x \in E$. Clearly, linear operators are 1-homogeneous.

2.1. Quasi-Banach function lattices. A quasi-Banach function lattice (lattice for short) $\left(X,\|\cdot\|_{X}\right)$ over $(\Omega, \Sigma, \mu)$ (over $\mu$ for short) is defined to be a subspace of $L^{0}(\mu)$, endowed with a complete quasi-norm $\|\cdot\|_{X}$ and such that there exists $h \in X$ with $h>0$ a.e., and if $f \in L^{0}$ satisfies $|f| \leq|g|$ a.e. for some $g \in X$, then $f \in X$ and $\|f\|_{X} \leq\|g\|_{X}$. 
A quasi-Banach function lattice $X$ on $(\Omega, \Sigma, \mu)$ is said to be order continuous if for every non-negative sequence $f_{n} \downarrow 0$ a.e. it holds that $\left\|f_{n}\right\|_{X} \rightarrow 0$.

The Köthe dual space $X^{\prime}$ of a Banach function lattice $X$ over $(\Omega, \Sigma, \mu)$ is defined as the space of all $f \in L^{0}(\mu)$ such that $\int_{\Omega}|f g| d \mu<\infty$ for every $g \in X$. It is a Banach function lattice over $(\Omega, \Sigma, \mu)$ when equipped with the norm

$$
\|f\|_{X^{\prime}}=\sup _{\|g\|_{X} \leq 1} \int_{\Omega}|f g| d \mu \text {. }
$$

It is well known that a Banach lattice $X$ is order continuous if and only if the map $X^{\prime} \ni g \mapsto x_{g}^{*} \in X^{*}$ given by $x_{g}^{*}(f)=\int_{\Omega} f g d \mu$ for all $f \in X$ is an order isometric isomorphism of $X^{\prime}$ onto $X^{*}$ (see, e.g., [21]). This fact motivates to identify $X^{\prime}$ with the subspace of the Banach dual space $X^{*}$ defined by the so called integral functionals - the ones given by the shown formula.

An important class of Banach lattices are the so called rearrangement invariant spaces (r.i. for short). We recall the definition; given $f \in L^{0}(\mu)$, its distribution function is defined by $\mu_{f}(t):=\mu(\{\omega \in \Omega:|f(\omega)|>t\}), t \geq 0$. Then a quasiBanach function lattice $X$ over $(\Omega, \Sigma, \mu)$ is said to be a r.i. space whenever $g \in X$ and $\|f\|_{X}=\|g\|_{X}$ provided $\mu_{f}=\mu_{g}$ and $f \in X$. Clearly for a r.i. quasi-Banach space $X,\left\|\chi_{A}\right\|_{X}$ depends only on $\mu(A)$ for any measurable set $A$ of finite measure. If $(\Omega, \Sigma, \mu)$ is a nonatomic measure space, then for every $t \in \mathbb{R}_{+}$with $t \leq \mu(\Omega)$, we may define the function $\phi_{X}$ by $\phi_{X}(t)=\left\|\chi_{A}\right\|_{X}$, where $A$ is any measurable set with $\mu(A)=t$. This function is called the fundamental function of $X$. For every r.i. Banach space $X$ on a non-atomic measure space $(\Omega, \Sigma, \mu)$ we have (see [1]),

$$
\phi_{X}(t) \phi_{X^{\prime}}(t)=t, \quad 0 \leq t<\mu(\Omega) .
$$

Important examples of r.i. spaces are Lorentz spaces, Marcinkiewicz spaces and Orlicz spaces. Let $(\Omega, \Sigma, \mu)$ be a measure space and let $0<p, q<\infty$. The Lorentz (resp., Marcinkiewicz) space $L_{p, q}=L_{p, q}(\mu)$ (resp., $L_{p, \infty}=L_{p, \infty}(\mu)$ ) is defined to be the r.i. quasi-Banach space of all measurable functions $f \in L^{0}(\mu)$ such that the following quasi-norm is finite:

$$
\|f\|_{p, q}=\left(\int_{0}^{\infty}\left(t^{\frac{1}{p}-\frac{1}{q}} f^{*}(t)\right)^{q} d t\right)^{1 / q}
$$

(resp.,

$$
\left.\|f\|_{p, \infty}=\sup _{0<t<\mu(\Omega)} t^{1 / p} f^{*}(t)\right) .
$$

It is well known that $L_{p, q}$ is normable whenever $1<p<\infty$ and $1 \leq q \leq \infty$. More precisely, $\left(L_{p, q},\|\cdot\|_{p, q}^{\prime}\right)$ is a r.i. Banach space equipped with the norm $\|\cdot\|_{p, q}^{\prime} \equiv\|\cdot\|_{p, q}$ given by

$$
\|f\|_{p, q}^{\prime}:=\left(\int_{0}^{\infty}\left(t^{\frac{1}{p}-\frac{1}{q}} f^{* *}(t)\right)^{q} d t\right)^{1 / q}, \quad f \in L_{p, q},
$$

(with obvious modification for $q=\infty$ ) where $f^{* *}(t)=\frac{1}{t} \int_{0}^{t} f^{*}(s) d s$. 
The Orlicz space $L_{\Phi}(\mu)$ is generated by the Luxemburg norm

$$
\|f\|_{\Phi}=\inf \left\{\lambda>0: \int_{\Omega} \Phi(|f| / \lambda) d \mu \leq 1\right\}
$$

where $\Phi$ is an Orlicz function, that is, an increasing convex function on $[0, \infty)$ such that $\Phi(0)=0$.

In the paper we present some results concerning so-called mixed spaces. Fix two measure spaces $\left(\Omega_{1}, \Sigma_{1}, \mu_{1}\right)$ and $\left(\Omega_{2}, \Sigma_{2}, \mu_{2}\right)$ and let $\left(\Omega=\Omega_{1} \times \Omega_{2}, \Sigma=\Sigma_{1} \times\right.$ $\left.\Sigma_{2}, \mu=\mu_{1} \times \mu_{2}\right)$ be the product measure space. Let $X$ be a function lattice on $\left(\Omega_{1}, \Sigma_{1}, \mu_{1}\right)$ and $Y$ a Banach function lattice on $\left(\Omega_{2}, \Sigma_{2}, \mu_{2}\right)$. The norm in $Y$ is said to be $\mu_{1}$-measurable if for any $\Sigma$-measurable function $f$ the function $v_{f}$ given by $v_{f}(s)=\|f(s, \cdot)\|_{Y}$ for all $s \in \Omega_{1}$ is $\mu_{1}$-measurable (we put $\|f(s, \cdot)\|_{Y}=+\infty$ if $f(s, \cdot) \notin Y)$. We note that if the measure $\mu_{1}$ is discrete or $\mu_{1}$ is arbitrary, and $Y$ has the weak Fatou property, then the norm in $Y$ is $\mu_{1}$-measurable.

In what follows if the norm in $Y$ is $\mu_{1}$-measurable we denote by $X[Y]$ the mixed space to be the Banach function lattice of all $f \in L^{0}(\mu)$ such that $v_{f} \in X$, equipped with the norm $\|f\|=\|\| f(\cdot, t)\left\|_{Y}\right\|_{X}$.

Given quasi-Banach lattices $X$ and $Y$ over $(\Omega, \Sigma, \mu)$, we define the space of multiplication operators $M(X, Y)$ to be the space of all functions in $L^{0}(\mu)$ defining (bounded and linear) operators from $X$ to $Y$ by pointwise multiplication, equipped with the operator quasi-norm.

Convexity and concavity of Banach function lattices will play a key role in our study. We recall that for $0<p<\infty$, the Banach function lattice $(X,\|\cdot\|)$ is $p$-convex, respectively, $p$-concave, if there are positive constants $C^{(p)}$ and $C_{(p)}$ such that

$$
\left\|\left(\sum_{i=1}^{n}\left|f_{i}\right|^{p}\right)^{1 / p}\right\|_{X} \leq C^{(p)}\left(\sum_{i=1}^{n}\left\|f_{i}\right\|_{X}^{p}\right)^{1 / p}
$$

respectively,

$$
\left(\sum_{i=1}^{n}\left\|f_{i}\right\|_{X}^{p}\right)^{1 / p} \leq C_{(p)}\left\|\left(\sum_{i=1}^{n}\left|f_{i}\right|^{p}\right)^{1 / p}\right\|_{X}
$$

for every choice of elements $f_{1}, \ldots f_{n} \in X$. The least constant $C^{(p)}$ (resp., $\left.C_{(p)}\right)$ is denoted by $M^{(p)}(X)$ (resp., $\left.M_{(p)}(X)\right)$.

If $X$ is a quasi-Banach function lattice and $0<p<\infty$, then the $p$-th power of $X$ is defined as

$$
X^{p}:=\left\{f \in L^{0}(\mu):|f|^{1 / p} \in X\right\} .
$$

This is a quasi-Banach function lattice over $\Omega$ when endowed with the quasi-norm $\|f\|_{X^{p}}:=\left\||f|^{1 / p}\right\|_{X}^{p}, f \in X^{p}$, and a Banach space whenever $X$ is $p$-convex (with a norm that is equivalent to $\left.\|\cdot\|_{X^{p}}\right)$. More precisely, $\|\cdot\|_{X^{p}}$ defines a norm if and only if $X$ is $p$-convex with $p$-convexity constant 1 . Note also that $X$ is order continuous if and only if $X^{p}$ is so for any/all $0<p<\infty$. 
Throughout the paper, given a quasi-Banach space $E$ and a quasi-Banach function lattice $X$ over $(\Omega, \Sigma, \mu)$, we define the quasi-Banach space $X(\mu, E)(X(E)$ for short) to be the space of all strongly measurable functions $f: \Omega \rightarrow E$ (i.e., $f$ is the limit in $E$ of a sequence of simple measurable functions) such that the function $\Omega \ni \omega \mapsto\|f(\omega)\|_{E} \in X$ equipped with the quasi-norm (norm provided $E$ and $X$ are Banach spaces) $\|f\|_{X(E)}=\|\| f(\cdot)\left\|_{E}\right\|_{X}$.

2.2. Groups acting on lattices. We recall briefly the basic facts which we will use. A topological group is a group with a topology such that the maps $(x, y) \mapsto x y$ and $x \mapsto x^{-1}$ are continuous.

Let $G$ be a locally compact group. If $x, y \in G$ and $B \subset G$, we write $x y$ for the multiplication of both elements and $x B$ for the set defined by the (left) multiplication of $x$ by all the elements of $B$. Consider a left Haar measure on $G$, that is, a (positive) measure $\lambda$ on the $\sigma$-algebra $\mathcal{B}(G)$ of Borel sets of $G$ that is nonzero on all nonempty open sets and is left invariant, meaning that $\lambda(x B)=\lambda(B)$ for all Borel sets $B$ and all $x \in G$. If $\lambda$ is a left Haar measure on $G$, then $\rho(B):=\lambda\left(B^{-1}\right)$ defines a measure on $\mathcal{B}(G)$ which satisfies $\rho(B x)=\rho(B)$ for all $B \in \mathcal{B}(G)$ and all $x \in G$. Here as usual $B^{-1}=\left\{x^{-1}: x \in B\right\}$. The measure $\rho$ is called a right Haar measure on $G$. Left and right Haar measures are unique up to multiplication by positive constants. Throughout the paper we will consider normalized Haar measures.

A locally compact group $G$ is called unimodular if $\lambda=\rho$. It is well known that compact groups and Abelian groups are unimodular. The group $G$ is said to be amenable if for every compact subset $K$ in $G$ and every $\varepsilon>0$ there exists a compact $U \subset G$ with $\lambda(K U) \leq(1+\varepsilon) \lambda(U)$. We remark that both commutative and compact groups are amenable.

Let $(\Omega, \Sigma, \mu)$ be a measure space. We say that the group $G$ with left Haar measure $\lambda$ acts transitively on $\Omega$ (the group $G$ acts on $\Omega$ for short) if there is an operation $G \times \Omega \ni(x, \omega) \mapsto x \diamond \omega \in \Omega$ such that $(x y) \diamond \omega=x \diamond(y \diamond \omega), 1 \diamond \omega=\omega$ and $G \diamond \omega=\Omega$ for all $x, y \in G$ and $\omega \in \Omega$.

The measure $\mu$ is called $G$-invariant if the group action is also measurable and $\mu(x \diamond A)=\mu(A)$ for all $x \in G$ and all $A \in \Sigma$; in this case we say that $G$ acts invariantly over $(\Omega, \Sigma, \mu)$.

The construction explained above involves two different actions: the multiplication on the group $G$ and the action of the group in a measure space. In most of the cases we are interested in, both structures coincide, in the sense that the measure space on which $G$ acts is $G$ itself. However, this is not necessarily the case.

Now we present some examples; the first two ones are relevant for the rest of the paper since in general a group acts on itself in two different ways. 
Example 2.1.

(1) Every locally compact group $G$ acts on itself by $(x, y) \mapsto x \diamond y:=x y$. We will say then that $G$ acts from the left to itself. Clearly, the left Haar measure is $G$-invariant with respect to this action.

(2) Every locally compact group $G$ acts also on itself by $(x, y) \mapsto x \diamond y:=y x^{-1}$. When we consider this action we will say that $G$ acts from the right on itself. The right Haar measure is $G$-invariant with respect to this action.

(3) The next example is more concrete than the other ones; our aim is to show that our construction may provide results in different scenarios. The group $S O(n)$ of orthogonal matrices of rank $n, n \geq 2$ acts on the $(n-1)$ dimensional unit sphere $\mathbb{S}^{n-1}$ by $(A, x) \mapsto A x$. This allows us to identify the classical notions of rotation invariant spaces and operators with our definition of $S O(n)$-invariant spaces and operators.

2.3. Translation invariance. If $x \in G$, we denote the translation of functions $f \in L^{0}(\Omega)$ by $x$ as $\tau_{x}$, i.e.,

$$
\tau_{x} f(\omega):=f(x \diamond \omega), \quad \omega \in \Omega .
$$

A quasi-Banach function lattice $X$ over $\Omega$ is said to be weak-G-invariant if $\tau_{x}(f) \in$ $X$ for all $f \in X$ and $x \in G$. If we also have $\left\|\tau_{x}(f)\right\|=\|f\|$, then $X$ is called $G$-invariant.

Assume now that $G$ acts on two measure spaces with the measures $\mu$ and $\nu$, respectively. A map $\Phi: X(\mu) \rightarrow Y(\nu)$ between weak- $G$-invariant quasi-Banach function spaces $X(\mu)$ and $Y(\nu)$ is called translation $G$-invariant if $\Phi \circ \tau_{x}=\tau_{x} \circ \Phi$ for all $x \in G$.

In the case of spaces of vector-valued functions, an operator $\Phi: X(\mu, E) \rightarrow$ $Y(\nu, F)$ is said to be $G$-invariant if for all $x \in G$ we have

$$
\left\|\tau_{x}(\Phi f)(\cdot)\right\|_{F}=\left\|\Phi\left(\tau_{x} f\right)(\cdot)\right\|_{F}, \quad x \in G .
$$

In what follows, if $G$ is a locally compact group with left Haar measure $\lambda$ (resp., right Haar measure $\rho), E$ is a quasi-Banach space and $X$ is a quasi-Banach function lattice on $(G, \lambda)$ (resp., $(G, \rho)$ we will write $X(\lambda, E)$ (resp., $X(\rho, E))$ instead of $X(E)$ or $X(G, E)$ for the aim of clarity.

\section{Abstract convolution}

Below we explain the main tool of the present paper, that consists of a generalized convolution of functions with functions, as well as more generally functions with functionals. Consider a quasi-Banach function lattice $X$ over $(\Omega, \Sigma, \mu)$, and a locally compact group $G$ with the left Haar measure $\lambda$ acting transitively on $\Omega$ by the action $\diamond$. Given two nonnegative measurable functions $g: G \rightarrow \mathbb{R}$ and $h: \Omega \rightarrow \mathbb{R}$, 
we define $g \circledast h: \Omega \rightarrow[0, \infty]$ by

$$
(g \circledast h)(\omega):=\int_{G} g(x) h\left(x^{-1} \diamond \omega\right) d \lambda(x) ;
$$

note that the function $g \circledast h$ by the Fubini-Tonelli theorem (on iterated integrals) is measurable. If we want to stress the action $\diamond$ of $G$ on $\Omega$, then we write $g \circledast_{\diamond} h$ instead of $g \circledast h$.

In what follows we will need the following elementary observation.

Proposition 3.1. Let $G$ be a locally compact group with left Haar measure $\lambda$. Consider the action of $G$ on itself from the right (as shown in Example 2.1(2)), and define $g \circledast h$ with respect to this action.

(i) Suppose that $X$ is a $G$-invariant Banach function space over $(G, \lambda)$ with respect to the left action of the group on itself. Then for two nonnegative functions $g \in X$ and $h \in X^{\prime}$

$$
\|g \circledast h\|_{L^{\infty}(\lambda)} \leq\|g\|_{X}\|h\|_{X^{\prime}} .
$$

(ii) For every nonnegative $f \in L_{1}(\lambda)$ and $y \in G$,

$$
\left(\chi_{G} \circledast f\right)(y)=\|f\|_{L_{1}(\lambda)} .
$$

Proof. (i) For every $y \in G$,

$$
\begin{aligned}
|(g \circledast h)(y)| & =\left|\int_{G} g(x) h\left(x^{-1} \diamond y\right) d \lambda(x)\right| \\
& =\left|\int_{G} g(x) h(y x) d \lambda(x)\right|=\left|\int_{G} g\left(y^{-1} x\right) h(x) d \lambda(x)\right| \\
& \leq\left\|\tau_{y^{-1}}(g)\right\|_{X}\|h\|_{X^{\prime}}=\|g\|_{X}\|h\|_{X^{\prime}},
\end{aligned}
$$

and so the required estimate follows. (ii) Clearly, $L^{1}(\lambda)$ is $G$-invariant with respect to the left action, hence for every $y \in G$ as desired

$$
\left(\chi_{G} \circledast f\right)(y)=\int_{G}\left|f\left(x^{-1} \diamond y\right)\right| d \lambda(x)=\int_{G}|f(y x)| d \lambda(x)=\|f\|_{L^{1}(\lambda)} .
$$

This completes the proof.

We also need to convolute functions with functionals, and start with an informal idea of what we aim at. Given $g \in L^{0}(\lambda)$ and $\varphi \in X^{*}$ we define the abstract convolution $g \star \varphi: X \rightarrow \mathbb{R}$ of the function $g$ with the functional $\varphi$ by

$$
(g \star \varphi)(f):=\int_{G} g(x) \varphi(f(x \diamond \cdot)) d \lambda(x), \quad f \in X
$$

whenever the integral make sense. We show below that this definition coincides with the previously defined convolution of functions if $\varphi$ is an integral functional. 
Remark 3.2. Assume that $X$ is a $G$-invariant Banach function lattice, the function $G \ni x \mapsto \varphi(f(x \diamond \cdot))$ is measurable for all $f \in X$, and $g \in L_{1}(\lambda)$. Then $g \star \varphi \in X^{*}$ and $\|g \star \varphi\|_{X^{*}} \leq\|g\|_{1}\|\varphi\|_{X^{*}}$. Indeed,

$$
\begin{aligned}
\int_{G}|g(x) \varphi(f(x \diamond \cdot))| d \lambda(x) & \leq \int_{G} \mid g(x)\|\| \varphi\left\|_{X^{*}}\right\| f(x \diamond \cdot) \|_{X} d \lambda(x) \\
& \leq\|g\|_{1}\|\varphi\|_{X^{*}}\|f\|_{X} .
\end{aligned}
$$

Now let us explain the two main cases we are going to consider: the first one involving order continuous Banach function spaces $X$ as domain spaces of the functionals $\varphi$, and the second one for functionals $\varphi$ on Banach spaces of continuous functions on a compact set.

3.1. Order continuous case. Recall that if $X$ is an order continuous Banach function lattice over $\Omega$, $\mu$, then every $\varphi \in X^{*}$ has a density $h^{\prime} \in X^{\prime}$, i.e., $\varphi(f)=$ $\int_{\Omega} f h d \mu$ for every $f \in X$.

Lemma 3.3. Let $G$ be a locally compact group with left Haar measure $\lambda$ acting transitively on $(\Omega, \Sigma, \mu)$ by the action $\diamond$, and let $X$ be a $G$-invariant Banach function lattice $X$ over $(\Omega, \Sigma, \mu)$. Then for every $g \in L_{1}(\lambda)$ and $\varphi \in X^{*}$ with density $h \in X^{\prime}$ we have that

$$
g \star \varphi \in X^{*}
$$

with density $g \circledast_{\diamond} h \in X^{\prime}$ and $\|g \star \varphi\| \leq\|g\|_{1}\|h\|_{X^{\prime}}$.

Proof. Take some $f \in X$. The $G$-invariance of $X$ shows that for every $x \in G$

$$
\int_{\Omega}|f(x \diamond \omega) h(\omega)| d \mu(\omega) \leq\|f(x \diamond \cdot)\|_{X}\|h\|_{X^{\prime}}=\|f\|_{X}\|h\|_{X^{\prime}} .
$$

Since $g$ is integrable, the iterated integral

$$
\int_{G}\left(\int_{\Omega} f(x \diamond \omega) h(\omega) d \mu(\omega)\right) g(x) d \lambda(x)
$$

exists, and moreover by the translation invariant action of $G$ on $\Omega$ it equals

$$
\int_{G}\left(\int_{\Omega} h\left(x^{-1} \diamond \omega\right) f(\omega) d \mu(\omega)\right) g(x) d \lambda(x) .
$$

Hence the Fubini-Tonelli theorem implies

$$
\begin{aligned}
\int_{\Omega} f(\omega) g \circledast h(\omega) d \mu(\omega) & =\int_{G} g(x)\left(\int_{\Omega} h\left(x^{-1} \diamond \omega\right) f(\omega) d \mu(\omega)\right) d \lambda(x) \\
& =\int_{G} g(x)\left(\int_{\Omega} h(\omega) f(x \diamond \omega) d \mu(\omega)\right) d \lambda(x) \\
& =\int_{G} g(x) \varphi(f(x \diamond \cdot)) d \lambda(x)=(g \star \varphi)(f),
\end{aligned}
$$

and so the above inequality yields the statement. 
3.2. Non-order continuous case. Let $G$ be a compact group with left Haar measure $\lambda$ (recall that then the group is unimodular), and $X=C(G)$. In what follows we consider the left action of the group on itself, that is $x \diamond \omega=x \omega$. Then $C(G)$ of course is $G$-invariant(note that now $x$ and $\omega$ are both elements of $G$, so we write $y$ instead of $\omega$ to emphasise this fact).

Take $\varphi \in C(G)^{*}$ and $g \in L_{1}(\lambda)$, both nonnegative and of norm one. By Riesz's Theorem, there is a Borel probability measure $P$ on $G$ such that $\varphi(f):=\int_{G} f d P$ for all $f \in C(G)$. Then for every $f \in C(G)$ the real-valued function

$$
G \ni x \mapsto \varphi(f(x \diamond \cdot))=\int_{G} f(x y) d P(y)
$$

is Borel-measurable. Hence $g \star \varphi \in C(G)^{*}$ with norm $\|g\|_{1}\|\varphi\|=1$ by Remark 3.2 (take $f=1$ to see that we in fact have equality). Moreover, by Fubini's theorem

$$
\begin{aligned}
(g \star \varphi)(f) & =\int_{G} g(x) \varphi(f(x \diamond \cdot)) d \lambda(x) \\
& =\int_{G} g(x)\left(\int_{G} f(x y) d P(y)\right) d \lambda(x) \\
& =\int_{G}\left(\int_{G} g\left(x y^{-1}\right) f(x) d \lambda(x)\right) d P(y) \\
& =\int_{G} f(x)\left(\int_{G} g\left(x y^{-1}\right) d P(y)\right) d \lambda(x),
\end{aligned}
$$

which shows that $g \star \varphi$ is a $\lambda$-continuous Borel probability on $G$ with the density

$$
h(x)=\int_{G} g\left(x y^{-1}\right) d P(y), \quad x \in G .
$$

We summarize these facts in the following

Lemma 3.4. Let $\varphi \in C(G)^{*}$ and $g \in L_{1}(\lambda)$, both nonnegative and of norm 1 . Then $g \star \varphi$ is a $\lambda$-continuous Borel probability measure on $G$.

\section{Matching lattices}

We now introduce a key definition in our study. Let $G$ be a locally compact group with left Haar measure $\lambda$, and let $X$ and $Y$ be Banach function lattices over $(G, \lambda) . \quad X$ is said to match $Y$ if there exists a constant $c>0$ such that for all compact sets $K \subset G$ there are nonnegative functions $g \in X$ and $h \in S_{Y^{\prime}}$ such that $\|g\|_{X} \leq c$ and $g \circledast h(x)=1$ for all $x \in K$.

Notice that the group action of $G$ on itself here may be, either the right or the left one.

We point out that the just introduced notion is inspired by [19, Lemma 3] of Herz and Riviére. In our language the authors prove that $L_{p}(\lambda)$ matches $L_{p}(\lambda)$ provided $G$ is an amenable group $G$. We now generalize this result. 


\subsection{Mixed spaces.}

Lemma 4.1. Let $G_{1}$ and $G_{2}$ be amenable, locally compact groups with nonatomic (left) Haar measures $\lambda_{1}$ and $\lambda_{2}$ and let $X_{1}, Y_{1}$ and $X_{2}, Y_{2}$ be r.i. Banach function spaces over $\lambda_{1}$ and $\lambda_{2}$, respectively. If both norms in $X_{2}$ and $Y_{2}$ are $\lambda_{1}$-measurable, then $X_{1}\left[X_{2}\right]$ matches the mixed space $Y_{1}\left[Y_{2}\right]$ with respect to the right action of $G_{1} \times G_{2}$ on itself whenever the fundamental functions satisfy $\phi_{X_{1}}(s) \phi_{X_{2}}(t)=$ $\phi_{Y_{1}}(s) \phi_{Y_{2}}(t)$ for all $s \in\left(0, \lambda_{1}\left(G_{1}\right)\right), t \in\left(0, \lambda_{2}\left(G_{2}\right)\right)$.

Proof. Obviously, it suffices to show the assertion for compact sets $K=K_{1} \times K_{2} \subset$ $G_{1} \times G_{2}$. Let us fix compact sets $U_{1} \subset G_{1}$ and $U_{2} \subset G_{2}$ such that

$$
\lambda_{j}\left(K_{j}^{-1} U_{j}\right) \leq 2 \lambda_{j}\left(U_{j}\right), \quad j=1,2 .
$$

Since $\phi_{X_{j}}$ are quasi-concave functions, $\phi_{X_{j}}(t) \leq 2 \phi_{X_{j}}(t / 2)$ for all $t \in\left(0, \lambda_{j}\left(G_{j}\right)\right)$ $(j=0,1)$. Then we get for $U=U_{1} \times U_{2}$,

$$
\begin{aligned}
\left\|\chi_{K^{-1} U}\right\|_{X_{1}\left[X_{2}\right]} & =\|\| \chi_{K_{1}^{-1} U_{1} \times K_{2}^{-1} U_{2}}\left\|_{X_{2}}\right\|_{X_{1}} \\
& =\phi_{X_{1}}\left(\lambda_{1}\left(K_{1}^{-1} U_{1}\right)\right) \phi_{X_{2}}\left(\lambda_{2}\left(K_{2}^{-1} U_{2}\right)\right) \\
& \leq 4 \phi_{X_{1}}\left(\lambda_{1}\left(U_{1}\right)\right) \phi_{X_{2}}\left(\lambda_{2}\left(U_{2}\right)\right) .
\end{aligned}
$$

Therefore, for $g:=\phi_{X_{1}}\left(\lambda_{1}\left(U_{1}\right)\right)^{-1} \phi_{X_{2}}\left(\lambda_{2}\left(U_{2}\right)\right)^{-1} \chi_{K^{-1} U}$, we obtain $\|g\|_{X_{1}\left[X_{2}\right]} \leq 4$.

Applying the well-known Köthe duality formula $\left(Y_{1}\left[Y_{2}\right]\right)^{\prime}=Y_{1}^{\prime}\left[Y_{2}^{\prime}\right]$ (with equality of norms), we get that

$$
\left\|\chi_{U}\right\|_{\left(Y_{1}\left[Y_{2}\right]\right)^{\prime}}=\left\|\chi_{U}\right\|_{Y_{1}^{\prime}\left[Y_{2}^{\prime}\right]}=\phi_{Y_{1}^{\prime}}\left(\lambda_{1}\left(U_{1}\right)\right) \phi_{Y_{2}^{\prime}}\left(\lambda_{2}\left(U_{2}\right)\right)
$$

and so $h:=\phi_{Y_{1}^{\prime}}\left(\lambda_{1}\left(U_{1}\right)\right)^{-1} \phi_{Y_{2}^{\prime}}\left(\lambda_{1}\left(U_{2}\right)\right)^{-1} \chi_{U}$ belongs to the unit sphere of $\left(Y_{1}\left[Y_{2}\right]\right)^{\prime}$.

Fix now $x \in K$ and let $G:=G_{1} \times G_{2}, \lambda:=\lambda_{1} \times \lambda_{2}$. Then we have (use Example $2.1(2))$ that

$$
\begin{aligned}
g \circledast h(x) & \left.=\int_{G} g(y) h(x y) d \lambda(y) \leq \phi_{X_{1}}\left(\lambda_{1}\left(U_{1}\right)\right)^{-1} \phi_{Y_{1}^{\prime}}\left(\lambda_{1}\left(U_{1}\right)\right)\right)^{-1} \\
& \times \phi_{X_{2}}\left(\lambda_{2}\left(U_{2}\right)\right)^{-1} \phi_{Y_{2}^{\prime}}\left(\lambda_{2}\left(U_{2}\right)\right)^{-1} \int_{G} \chi_{K^{-1} U}\left(x^{-1} y\right) \chi_{U}(y) d \lambda(y) \\
& =\frac{\lambda_{1}\left(U_{1}\right)}{\phi_{X_{1}}\left(\lambda_{1}\left(U_{1}\right)\right) \phi_{Y_{1}^{\prime}}\left(\lambda_{1}\left(U_{1}\right)\right)} \frac{\lambda_{2}\left(U_{2}\right)}{\phi_{X_{2}}\left(\lambda_{2}\left(U_{2}\right)\right) \phi_{Y_{2}^{\prime}}\left(\lambda_{2}\left(U_{2}\right)\right)} .
\end{aligned}
$$

Our hypotheses on the fundamental functions imply that the last expression is equal to 1 . We conclude that the requirements in the definition of matching are satisfied for $g$ and $h$, and so this completes the proof.

An immediate consequence of the above lemma is the following corollary.

Corollary 4.2. Let $1 \leq p_{1}, p_{2}, q_{1}, q_{2}<\infty$ and $G_{1}, G_{2}$ be amenable, locally compact groups with (left) Haar measures $\lambda_{1}$ and $\lambda_{2}$. Then the mixed space $L_{p_{1}}\left(\lambda_{1}\right)\left[L_{p_{2}}\left(\lambda_{2}\right)\right]$ 
matches the mixed space $L_{q_{1}}\left(\lambda_{1}\right)\left[L_{q_{2}}\left(\lambda_{2}\right)\right]$ with respect to the right action of $G_{1} \times G_{2}$ on itself whenever $1 / p_{1}+1 / p_{2}=1 / q_{1}+1 / q_{2}$.

\subsection{Rearrangement invariant spaces.}

Lemma 4.3. Let $G$ be an amenable locally compact group with nonatomic left Haar measure $\lambda$. If $X$ and $Y$ are r.i. Banach function spaces over $\lambda$ such that $\phi_{X}=\phi_{Y}$, then $X$ matches $Y$ with respect to the right action of $G$ on itself.

Proof. Recall that for the case of Banach function spaces, $\phi_{X}(s) \phi_{X^{\prime}}(s)=s$ for all $s \in(0, \lambda(G))$. Since $\phi_{X}$ is quasi-concave, $\phi(t) \leq 2 \phi_{X}(t / 2)$ for all $t \in(0, \lambda(G))$.

Now we use that $G$ is amenable. Consider a compact subset $K$, and a compact subset $U$ of $G$ such that $\lambda\left(K^{-1} U\right) \leq 2 \lambda(U)$. Then

$$
\left\|\chi_{K^{-1} U}\right\|_{X}=\phi_{X}\left(\lambda\left(K^{-1} U\right)\right) \leq 2 \phi_{X}\left(\lambda\left(K^{-1} U\right) / 2\right) \leq 2 \phi_{X}(\lambda(U)) .
$$

Let $g=\frac{1}{\phi_{X}(\lambda(U))} \chi_{K^{-1} U}$ and $h=\frac{1}{\phi_{Y^{\prime}}(\lambda(U))} \chi_{U}$. Then

$$
\|g\|_{X}=\frac{1}{\phi_{X}(\lambda(U))}\left\|\chi_{K^{-1} U}\right\|_{X} \leq 2
$$

and

$$
\|h\|_{Y^{\prime}}=\frac{\phi_{Y^{\prime}}(\lambda(U))}{\phi_{Y^{\prime}}(\lambda(U))}=1
$$

Our hypothesis is equivalent to $\phi_{X^{\prime}}=\phi_{Y^{\prime}}$, and so for all $x \in K$ we get (considering the right action of $G$ on itself)

$$
\begin{aligned}
(g \circledast h)(x) & =\frac{1}{\phi_{X}(\lambda(U)) \phi_{Y^{\prime}}(\lambda(U))} \int_{G} \chi_{K^{-1} U}\left(x^{-1} y\right) \chi_{U}(y) d \lambda\left(x^{-1} y\right) \\
& =\frac{1}{\phi_{X}(\lambda(U)) \phi_{Y^{\prime}}(\lambda(U))} \int_{G} \chi_{K^{-1} U}\left(x^{-1} y\right) \chi_{U}(y) d \lambda(y) \\
& =\frac{\lambda(U)}{\lambda(U)}=1 .
\end{aligned}
$$

This gives the result.

From this lemma we obtain examples of pairs of Lorentz spaces which match.

Corollary 4.4. Let $1<p<\infty, 1 \leq q_{1}, q_{2} \leq \infty$ and let $G$ be an amenable, locally compact group with nonatomic left Haar measure $\lambda$. Then the Lorentz space $L_{p, q_{1}}(\lambda)$ over $G$ matches the Lorentz space $L_{p, q_{2}}(\lambda)$ with respect to the right action of $G$ on itself.

Proof. Since $\left\|\chi_{A}\right\|_{p, q}=\lambda(A)^{1 / p}$ for all measurable $A \subset G, 1 \leq p, q \leq \infty$ where $0^{0}:=1$. Then the fundamental function coincides in both spaces (up to a constant $C$ ), and so Lemma 4.3 gives the result after renorming one of the spaces by this constant $C$. 


\section{Improving norm inequalities by convolution}

Let $\mathcal{X}$ and $\mathcal{Y}$ be Hausdorff topological vector spaces and let $X$ be a quasi-Banach space such that $X \hookrightarrow \mathcal{X}$ with continuous inclusion. If $T: X \rightarrow \mathcal{Y}$ is a continuous mapping and $X_{0}$ is a "test" subset, it is of powerful interest to study a class of quasi-Banach spaces $U$ and $V$ such that $X_{0} \subset U$ and $V \hookrightarrow \mathcal{Y}$ and $T$ is continuous from $\left(X_{0},\|\cdot\|_{U}\right)$ to $V$. In this case we say that $T$ extends from $U$ to $V$ on a test set $X_{0}$. If $T$ has continuous extension from $U$ to $V$, then we say for short that $T$ extends from $U$ to $V$.

Let $E$ and $F$ be quasi-Banach spaces. In several areas of modern analysis we may find relevant maps from quasi-Banach spaces $U \hookrightarrow L^{0}(\mu, E)$ to $L^{0}(\nu, F)$, where $L^{0}(\mu, E)$ and $L^{0}(\nu, F)$ denote the spaces of strongly measurable $E$-valued ( $F$-valued) functions over the corresponding measure spaces. In the case of scalarvalued measurable functions, many important operators in harmonic analysis defined between couples of quasi-Banach spaces have continuous extensions to other couples of quasi-Banach spaces. We note here that it is well known that if the group $G$ is amenable, then every convolution operator of weak type $(2,2)$ is in fact of strong type $(2,2)$. The mentioned extension problem was studied by many authors (see $[6,5,18,23,28]$ ). In particular, in Colzani's paper [5] it is proved that if $G$ is a locally compact non-compact group equipped with the left Haar measure and $0<p<\infty, 0<q \leq \infty$, then every left or right translation invariant operator (in our terminology this means $G$-invariant) on the Lorentz space $L_{p, q}(G)$ is bounded in $L_{p}(G)$; that is, it can be defined as an operator from $L_{p}(G)$ to $L_{p}(G)$. We also mention here a remarkable result by Shternberg [33], which states that if $G$ is a compact group and $0<p<2$, then every translation invariant sublinear operator that maps the Marcinkiewicz space $L_{p, \infty}(G)$ to $L^{0}(G)$ extends from $L_{p}(G)$ to $L_{p}(G)$. This result was firstly conjectured by Pisier for translation invariant operators that are bounded on the Marcinkiewicz spaces $L_{p, \infty}(G)$ (see [30]).

In the case of particular locally compact groups, translation invariant operators have a special form. It is immediate that for the locally compact group $\mathbb{R}^{n}$ with the usual additive structure, convolution operators commute with translations. It is also well-known that the converse is true. More precisely, the following result holds: If $1 \leq p, q \leq \infty$ and $T: L_{p}\left(\mathbb{R}^{n}\right) \rightarrow L_{q}\left(\mathbb{R}^{n}\right)$ is a bounded linear operator that commutes with translations (notation: $T \in \mathcal{M}^{p, q}\left(\mathbb{R}^{n}\right)$ ), then there exists a unique tempered distribution $v$ such that $T(f)=f \star v$ for Schwartz functions $f$ (see, e.g., [17, Theorem 2.5.2]). It should also be noticed that $\mathcal{M}^{p, q}\left(\mathbb{R}^{n}\right)$ is non-trivial only in the case $1 \leq p \leq q \leq \infty$, and only in the cases $p=1, p=2$ and $p=\infty$ there are complete characterizations of the spaces $\mathcal{M}^{p, p}\left(\mathbb{R}^{n}\right)$ (see [17, pp. 139-141]). 
In this section we are interested in the study of translation invariant mappings in vector-valued function spaces ( $m$-homogeneous operators, polynomials and multilinear mappings) which extend to the scale of vector-valued $L_{r}$-spaces.

5.1. Homogeneous mappings. The following results are our main tools. The first one is based on some well-known separation arguments that are in the core of the Maurey-Rosenthal factorization theory for homogeneous maps; it can be proved by using a slight modification of [12, Theorem 3.2]. For the sake of completeness we include a proof.

Proposition 5.1. Let $0<r<\infty$ and $0<m<\infty$. Assume that $X(E)$ and $Y(F)$ are vector-valued quasi-Banach function spaces over measure spaces $(\Omega, \Sigma, \mu)$ and $(\Upsilon, \Xi, \nu)$, respectively. Assume that $X$ is $r$-convex with constant $M^{r}(X)=1$ and order continuous, and $Y$ is $r / m$-convex with constant $M^{r / m}(Y)=1$. Furthermore, let $\Phi: X(E) \rightarrow Y(F)$ be an m-homogeneous map such that for all $f_{1}, \ldots, f_{n} \in X(E)$,

$$
\left\|\left(\sum_{k=1}^{n}\left\|\Phi f_{k}(\cdot)\right\|_{F}^{r / m}\right)^{m / r}\right\|_{Y} \leq\left\|\left(\sum_{k=1}^{n}\left\|f_{k}(\cdot)\right\|_{E}^{r}\right)^{1 / r}\right\|_{X}^{m}
$$

Then for every nonnegative weight $h \in\left(Y^{r / m}\right)^{\prime}$ with $\|h\|_{\left(Y^{r / m}\right)^{\prime}}=1$ there exists a nonnegative weight $\omega \in L^{0}(\mu)$ with $\|\omega\|_{\left(X^{r}\right)^{\prime}} \leq 1$ such that for all $f \in X(E)$,

$$
\int_{\Upsilon}\|\Phi f(\cdot)\|_{F}^{r / m} h d \nu \leq \int_{\Omega}\|f(\cdot)\|_{E}^{r} \omega d \mu .
$$

Proof. Fix a nonnegative weight $h \in\left(Y^{r / m}\right)^{\prime}$ with $\|h\|_{\left(Y^{r / m}\right)^{\prime}}=1$. First we remark that our hypothesis on $r / m$-convexity of $Y$ yields,

$$
M\left(Y, L_{r / m}\right)^{r / m}=\left(Y^{r / m}\right)^{\prime}
$$

isometrically (see, e.g., [29, Proposition 2.29 (ii),(iv)]). Thus, we can consider the multiplication operator $M_{h^{m / r}}: Y \rightarrow L_{r / m}$, for which $\left\|M_{h^{m / r}}\right\|=1$. Define the mapping

$$
\Psi:=\left(M_{h^{m / r}} \otimes I d_{F}\right) \circ \Phi: X(E) \rightarrow L_{r / m}(F),
$$

change the scalar multiplication on $X(E)$ by $\lambda \circ u:=\lambda^{1 / m}$ for all $\lambda \geq 0, u \in X(E)$, and consider the homogeneous embeddings (in the sense of [8])

$$
r_{1}:(X(E), \circ) \rightarrow X^{m}, \quad f \mapsto\|f(\cdot)\|_{E}^{m}
$$

and

$$
r_{2}: L_{r / m}(F) \rightarrow L_{r / m}, \quad g \mapsto\|g(\cdot)\|_{F}
$$


Then $\Psi:(X(E), \circ) \rightarrow L_{r / m}(F)$ is 1-homogeneous, and for every choice of finitely many $f_{1}, \ldots, f_{n} \in X(E)$ we get that

$$
\begin{array}{r}
\left\|\left(\sum_{k=1}^{n}\left\|\Psi f_{k}(\cdot)\right\|_{F}^{r / m}\right)^{m / r}\right\|_{L_{r / m}}=\left\|M_{h^{m / r}}\left(\sum_{k=1}^{n}\left\|\left(\Phi f_{k}\right)(\cdot)\right\|_{F}^{r / m}\right)^{m / r}\right\|_{L_{r / m}} \\
\leq\left\|\left(\sum_{k=1}^{n}\left\|\Phi f_{k}(\cdot)\right\|_{F}^{r / m}\right)^{m / r}\right\|_{Y} \leq\left\|\left(\sum_{k=1}^{n}\left\|f_{k}(\cdot)\right\|_{E}^{r}\right)^{1 / r}\right\|_{X}^{m} .
\end{array}
$$

This shows that $\Psi$ satisfies all the assumptions of [8, Theorem 2] which yields nonnegative weights $w \in L^{0}(\mu)$ with $\|w\|_{\left(X^{r / m}\right)^{\prime}} \leq 1$ and $w_{1} \in L^{0}(\nu)$ with $\left\|w_{1}\right\|_{\infty} \leq$ 1 such that for all $f \in X(E)$ we have

$$
\int_{\Upsilon} \frac{\left\|h^{m / r} \Phi(f)(\cdot)\right\|_{F}^{r / m}}{w_{1}} d \nu \leq \int_{\Omega}\|f(\cdot)\|_{E}^{r} w d \mu
$$

Since $\left\|w_{1}\right\|_{\infty} \leq 1$, this inequality immediately gives the conclusion.

Although simple, the next result shows one the main tools of our paper: Convolution with proper functions preserves and sometimes even improves the weights in domination inequalities like in (5.2).

Lemma 5.2. Let $G$ be a locally compact group with left Haar measure $\lambda$ which acts invariantly on $(\Omega, \Sigma, \mu)$ and $(\Upsilon, \Xi, \nu)$. Let $E$ and $F$ be quasi-Banach spaces, and $X$ and $Y$ weak-G-invariant quasi-Banach function lattices over $(\Omega, \Sigma, \mu)$ and $(\Upsilon, \Xi, \nu)$, respectively.

Furthermore, for $0<r, s<\infty$ let $\Phi: X(E) \rightarrow Y(F)$ be a G-invariant map such that there exist nonnegative weights $w_{1} \in L^{0}(\mu), w_{2} \in L^{0}(\nu)$ satisfying for all $f \in X(\mu, E)$

$$
\int_{\Upsilon}\|\Phi f(\cdot)\|_{F}^{s} w_{2} d \nu \leq \int_{\Omega}\|f(\cdot)\|_{E}^{r} w_{1} d \mu
$$

Then for all nonnegative $g \in L^{0}(\lambda)$ and all $f \in X(E)$ we have

$$
\int_{\Upsilon}\|\Phi f(\cdot)\|_{F}^{s} g \circledast w_{2} d \nu \leq \int_{\Omega}\|f(\cdot)\|_{E}^{r} g \circledast w_{1} d \mu .
$$

Proof. First note that the involved spaces are weak $G$-invariant, and so for every $x \in G$ the functions $f(x \diamond \cdot)$ and $(\Phi f)(x \diamond \cdot)$ belong to $X(E)$ and $Y(F)$, respectively. 
Making use of Fubini's theorem twice we have

$$
\begin{array}{rl}
\int_{\Upsilon}\|\Phi f(\cdot)\|_{F}^{s} & g \circledast w_{2} d \nu \\
& =\int_{G}\left(\int_{\Upsilon}\|(\Phi f)(v)\|_{F}^{s} w_{2}\left(x^{-1} \diamond v\right) d \nu(v)\right) g(x) d \lambda(x) \\
& =\int_{G}\left(\int_{\Upsilon}\|(\Phi f)(x \diamond v)\|_{F}^{s} w_{2}(v) d \nu(v)\right) g(x) d \lambda(x) \\
& =\int_{G}\left(\int_{\Upsilon}\left\|\tau_{x}(\Phi f)(\cdot)\right\|_{F}^{s} w_{2} d \nu\right) g(x) d \lambda(x) \\
& =\int_{G}\left(\int_{\Upsilon}\left\|\Phi\left(\tau_{x} f\right)(\cdot)\right\|_{F}^{s} w_{2} d \nu\right) g(x) d \lambda(x) \\
& =\int_{G}\left(\int_{\Upsilon} \|\left(\Phi(f(x \diamond \cdot))(v) \|_{F}^{s} w_{2}(v) d \nu(v)\right) g(x) d \lambda(x)\right. \\
& \leq \int_{G} g(x)\left(\int_{\Omega}\|f(x \diamond \omega)\|_{E}^{r} w_{1}(\omega) d \mu(\omega)\right) d \lambda(x) \\
& =\int_{G} g(x)\left(\int_{\Omega}\|f(\omega)\|_{E}^{r} w_{1}\left(x^{-1} \diamond \omega\right) d \mu(\omega)\right) d \lambda(x) \\
& =\int_{\Omega}\|f(\cdot)\|_{E}^{r} g w_{1} d \mu,
\end{array}
$$

which is the inequality we required.

We consider now the case in which both measure spaces in the previous results coincide with the group $G$, that is, $(\Omega, \Sigma, \mu)=(\Upsilon, \Xi, \nu)=(G, \mathcal{B}(G), \lambda)$. For the aim of simplifying the presentation of the next results, let us introduce some notation.

We will say that the space $X(\lambda, E)$ is a (weak) right- $G$-invariant space if it is (weak) $G$-invariant when the right action of $G$ on itself is considered. We will use the expression (weak) left- $G$-invariant for the symmetric definition, considering the left action.

An $m$-homogeneous map from the weak right- $G$-invariant space $X(\lambda, E)$ to the weak left- $G$-invariant space $Y(\lambda, F)$ is right-to-left $G$-invariant when it is $G$ invariant for the right and left actions of the spaces $X(\lambda, E)$ and $Y(\lambda, F)$, respectively. Other combinations (like left-to-left, left-to-right and right-to-right) are defined in the same way.

Combining Proposition 5.1 with Lemma 5.2 we get the following two abstract factorization theorems for translation invariant $m$-homogeneous operators between Banach function lattices.

Theorem 5.3. Let $G$ be a locally compact group with left Haar measure $\lambda$ and let $E, F$ be quasi-Banach spaces. For $0<m, r<\infty$, let $X$ be an order continuous, $r$-convex, left-G-invariant and weak right-G-invariant quasi-Banach function lattice over $G$, and let $Y$ be an $r / m$-convex, weak left-G-invariant quasi-Banach function 
lattice over $G$. Suppose that $X^{r}$ matches $Y^{r / m}$ (the left action in $G$ is considered for the definition of $\circledast$ appearing in the matching property).

Then every m-homogeneous right-to-left $G$-invariant operator $\Phi: X(E) \rightarrow Y(F)$ extends to a bounded $m$-homogeneous operator from $L_{r}(E)$ to $L_{r / m}(F)$ provided that there is a constant $M>0$ such that for all choices of functions $f_{1}, \ldots, f_{n} \in X(E)$ we have

$$
\left\|\left(\sum_{k=1}^{n}\left\|\Phi f_{k}(\cdot)\right\|_{F}^{r / m}\right)^{m / r}\right\|_{Y} \leq M\left\|\left(\sum_{k=1}^{n}\left\|f_{k}(\cdot)\right\|_{E}^{r}\right)^{1 / r}\right\|_{X}^{m}
$$

Proof. We may assume that $M(X)^{(r)}=M(Y)^{(r / m)}=M=1$. Let $K$ be a compact subset of $G$. By definition there are $c>0$ (independent of $K$ ) and nonnegative functions $g \in X^{r}$ and $h \in\left(Y^{r / m}\right)^{\prime}$ such that $\|h\|_{\left(Y^{r / m}\right)^{\prime}}=1,\|g\|_{X^{r}} \leq c$ and $g \circledast h(x)=1$ for all $x \in K$ (the left action is considered in the definition of $\circledast$ ).

By Proposition 5.1 we find a nonnegative weight $w \in\left(X^{r}\right)^{\prime}$ with $\|w\|_{\left(X^{r}\right)^{\prime}} \leq 1$ and such that for all $f \in X(E)$ we have

$$
\int_{G}\|\Phi f(\cdot)\|_{F}^{r / m} h d \lambda \leq \int_{G}\|f(\cdot)\|_{E}^{r} w d \lambda .
$$

By Lemma 5.2 we may replace the weights by $g \circledast h$ and $g \circledast w$ preserving the inequality, that is, for all $f \in X(E)$ we have

$$
\int_{G}\|\Phi f(\cdot)\|_{F}^{r / m} g \circledast h d \lambda \leq \int_{G}\|f(\cdot)\|_{E}^{r} g \circledast w d \lambda .
$$

We use $g \circledast h=1$ in $K$ and Proposition 3.1(1) to get,

$$
\begin{aligned}
\int_{K}\|\Phi f(\cdot)\|_{F}^{r / m} d \lambda & \leq \int_{G}\|\Phi f(\cdot)\|_{F}^{r / m} g \circledast h d \lambda \\
& \leq \int_{G}\|f(\cdot)\|_{E}^{r} g \circledast w d \lambda \leq c \int_{G}\|f(\cdot)\|_{E}^{r} d \lambda .
\end{aligned}
$$

Since the last inequality holds for all compact sets $K$, we are done.

For compact groups we give a variant of Theorem 5.3. It shows that under certain assumptions the matching requirement can be removed in the statement of the theorem. Note that in this case the operator is assumed to be right-to-right $G$-invariant, in contrast with the previous result, in which the map is required to be right-to-left $G$-invariant. A detailed analysis of the corresponding proofs shows that this depends on the symmetry of the integrals that appear in the computations. Although the next results look like similar to the previous one, the computations are in fact different. The same comments apply to the results on polynomials, multilinear and linear maps of the following sections.

Theorem 5.4. Let $0<r, m<\infty$. Let $G$ be a compact group with Haar measure $\lambda$, $X$ an $r$-convex and order continuous Banach function lattice over $G$, and $Y$ an $r / m$ concave Banach function lattice over $G$. Moreover let $E, F$ be quasi-Banach spaces. 
Suppose that both lattices are weak right-G-invariant, and that $\Phi: X(E) \rightarrow Y(F)$ is a right-to-right $G$-invariant m-homogeneous operator satisfying (5.1).

Then $\Phi$ extends to a bounded m-homogeneous operator from $L_{r}(E)$ to $L_{r / m}(F)$.

Proof. By [12, Theorem 3.2] the requirements above imply that there are measurable nonnegative functions $\omega_{2}$ and $\omega_{1}$ such that for all $f \in X(E)$,

$$
\int_{\Upsilon}\|\Phi f(\cdot)\|_{F}^{r / m} \omega_{2}^{-1} d \lambda \leq \int_{\Omega}\|f(\cdot)\|_{E}^{r} \omega_{1} d \lambda .
$$

We can write $\omega_{2}+1$ instead of $\omega_{2}$ preserving the inequality. This assures that $\omega_{2}^{-1}$ belongs to $L^{1}(\lambda)$, taking into account that $G$ is compact and so $\lambda$ is a probability measure. We use Lemma 5.2 and Proposition 3.1(ii) to get

$$
\begin{aligned}
\left\|\omega_{2}^{-1}\right\|_{L^{1}(\lambda)} \int_{G}\|\Phi f(\cdot)\|_{F}^{r / m} d \lambda & =\int_{G}\|\Phi f(\cdot)\|_{F}^{r / m} \chi_{G} \circledast \omega_{2}^{-1} d \lambda \\
& \leq \int_{G}\|f(\cdot)\|_{E}^{r} \chi_{G} \circledast \omega_{1} d \lambda \\
& =\left\|\omega_{1}\right\|_{L^{1}(\lambda)} \int_{G}\|f(\cdot)\|_{E}^{r} d \lambda,
\end{aligned}
$$

which completes the proof.

Corollary 5.5. Let $G$ be a compact group with Haar measure $\lambda$, and $E, F$ two quasi-Banach spaces. Given $0<p, q, r<\infty$ let $\Phi$ be a right-to-right $G$-invariant $m$-homogeneous operator from $L_{q}(\lambda, E)$ into $L_{p}(\lambda, F)$ satisfying (5.1). Then $\Phi$ defines an $m$-homogeneous operator from $L_{r / m}(\lambda, E)$ to $L_{r}(\lambda, F)$.

Proof. Note that $\lambda$ is a probability measure, and so for $p_{1}=\min \{p, r / m\}$ and $q_{1}=\max \{q, r\}$, the inclusions $L_{p}(\lambda) \hookrightarrow L_{p_{1}}(\lambda)$ and $L_{q_{1}}(\lambda) \hookrightarrow L_{q}(\lambda)$ are contractions. This means that $\Phi$ can be considered as an operator from $L_{q_{1}}(\lambda, E)$ to $L_{p_{1}}(\lambda, F)$. Also, we have that $L_{q_{1}}(\lambda)$ is $r$-convex and $L_{p_{1}}(\lambda)$ is $r / m$-concave. Since the inequality (5.1) holds when the operator is considered between $L_{q}(\lambda, E)$ and $L_{p}(\lambda, F)$, it still holds whenever we consider it as an operator between $L_{q_{1}}(\lambda, E)$ and $L_{p_{1}}(\lambda, F)$. Since $G$ is compact, it is unimodular, and hence it can be seen easily that $L_{s}(\lambda), 0<s<\infty$ is $G$-invariant with respect to both actions of the group on itself. As a consequence Corollary 5.4 implies the result.

5.2. Polynomials. Let us show some particular examples of applications of Theorem 5.3 for the case of Banach space valued $m$-homogeneous polynomials.

At first recall that if $E_{1}, \ldots, E_{m}$ and $F$ are quasi-Banach spaces, then an $m$ linear mapping $T: E_{1} \times \cdots \times E_{m} \rightarrow F$ is said to be bounded whenever there exists a constant $C>0$ such that $\left\|T\left(x_{1}, \ldots, x_{m}\right)\right\|_{Y} \leq C\left\|x_{1}\right\|_{E_{1}} \ldots\left\|x_{m}\right\|_{E_{m}}$ for all $\left(x_{1}, \ldots, x_{m}\right) \in E_{1} \times \cdots \times E_{m}$. If $T$ acts in quasi-Banach function lattices, then it is called positive whenever the image of each $m$-tuple of nonnegative functions is again a nonnegative function. 
An $m$-homogeneous polynomial $P$ between quasi-Banach spaces $E$ and $F$ is the restriction of a bounded $m$-linear mapping $\varphi: \prod_{k=1}^{m} E \rightarrow F$ to the diagonal $\triangle=\{(x, \ldots, x): x \in E\}$, and its norm is given by

$$
\|P\|:=\sup _{x \in B_{E}}\|P(x)\|_{F} .
$$

If $P$ is defined between quasi-Banach function lattices $X$ and $Y$, then we call it positive whenever $P(x) \in Y$ is nonnegative for all $x \in X$. It can be easily shown that every positive $m$-homogeneous polynomial in quasi-Banach function lattices is bounded.

Theorem 5.6. Let $G$ be a locally compact group with left Haar measure $\lambda$. Let $X$ and $Y$ be left $G$-invariant Banach function lattices on $G$ such that $X$ is also order continuous. Let $1 \leq r<\infty$ and $m \in \mathbb{N}$ such that $r \geq m$, and suppose that $X^{r}$ matches $Y^{r / m}$ (with respect to the left action of the group). Assume also that $X$ is $r$-convex and $Y$ is $r / m$-convex.

Then every positive m-homogeneous polynomial $P: X \rightarrow Y$ that is right-toleft $G$-invariant, extends to a positive m-homogeneous polynomial $P: L_{r}(\lambda) \rightarrow$ $L_{r / m}(\lambda)$.

Proof. We may assume that $M^{(r)}(X)=M^{(r / m)}(Y)=\|P\|=1$. The arguments that are needed for the proof are similar to the ones used in the previous section. Let $K$ be a compact subset of $G$. Since $X^{r}$ matches $Y^{r / m}$, there is a constant $c>0$ (independent of $K$ ) and there are nonnegative functions $g \in X^{r}$ and $h \in S_{\left(Y^{r / m}\right)^{\prime}}$ such that $\|g\|_{X^{r}} \leq c$ and $g \circledast h=1$ on $K$ (the left action in $G$ is considered in the definition of $\circledast)$.

Notice that $\left(\left(Y^{r / m}\right)^{\prime}\right)^{m / r}=M\left(Y, L_{r / m}\right)$ (see the proof of Proposition 5.1). Consider the map $M_{h^{m / r}} \circ P: X \rightarrow L_{r / m}$; it is of course a positive $m$-homogeneous polynomial. Proposition 4.1 in [12] gives that for every finite sequence $\left\{x_{k}\right\}_{k=1}^{n}$ in $X$,

$$
\left\|\left(\sum_{k=1}^{n}\left|M_{h^{m / r}} \circ P x_{k}\right|^{r / m}\right)^{m / r}\right\|_{L_{r / m}} \leq \frac{m^{m}}{m !}\left\|\left(\sum_{k=1}^{n}\left|x_{k}\right|^{r}\right)^{1 / r}\right\|_{X}^{m} .
$$

Notice now that $M\left(L_{r / m}, L_{r / m}\right)=L_{\infty}$, and so [12, Corollary 3.3] gives that there are weights $w_{1}$ and $w_{2}$ such that $x \in X$

$$
\left(\int_{K} \frac{\left|M_{h^{m / r}} \circ P x\right|^{r / m}}{w_{2}} d \lambda\right)^{m / r} \leq \frac{m^{m}}{m !}\left(\int_{G}|x|^{r} w_{1} d \lambda\right)^{m / r},
$$

where $\left\|w_{1}\right\|_{\left(X^{r}\right)^{\prime}} \leq 1$, and $\left\|w_{2}^{m / r}\right\|_{L^{\infty}} \leq 1$; in particular, $\left\|w_{2}\right\|_{L^{\infty}} \leq 1$. Therefore,

$$
\int_{K}|P x|^{r / m} h d \lambda \leq \int_{K} \frac{|P x|^{r / m} h}{w_{2}} d \lambda \leq\left(\frac{m^{m}}{m !}\right)^{r / m} \int_{G}|x|^{r} w_{1} d \lambda .
$$

An application of Lemma 5.2 and Proposition 3.1 (taking into account that $P$ is $G$ invariant when the right action is considered in $X$ and the left action is considered 
in $Y$ ), gives for all $x \in X$

$$
\begin{aligned}
\int_{K}|P x|^{r / m} d \lambda & =\int_{K}|P x|^{r / m} g \circledast h d \lambda \\
& \leq\left(\frac{m^{m}}{m !}\right)^{r / m} \int_{G}|x|^{r} g \circledast w_{1} d \lambda \\
& \leq\left(\frac{m^{m}}{m !}\right)^{r / m}\left\|g \circledast w_{1}\right\|_{L^{\infty}} \int_{G}|x|^{r} d \lambda \\
& \leq\left(\frac{m^{m}}{m !}\right)^{r / m}\|g\|_{X^{r}}\left\|w_{1}\right\|_{\left(X^{r}\right)^{\prime}} \int_{G}|x|^{r} d \lambda \\
& \leq c\left(\frac{m^{m}}{m !}\right)^{r / m} \int_{G}|x|^{r} d \lambda .
\end{aligned}
$$

This clearly implies that $P$ extends to a positive $m$-homogeneous polynomial from $L_{r}(\lambda)$ to $L_{r / m}(\lambda)$.

In particular, taking into account that for $r \leq s, L^{s}$ is $r$-convex, $L^{s / m}$ is $r / m$ convex and $\left(L^{s}\right)^{r}=L^{s / r}=\left(L^{s / m}\right)^{r / m}$ and that by Lemma 4.1 (see also [19, Lemma 3]) $L^{s / r}$ matches with itself we get the following result.

Corollary 5.7. Let $G$ be an amenable locally compact group with left Haar measure $\lambda, 1 \leq r \leq s<\infty, m \in \mathbb{N}$ and $1 \leq r / m$. Let $P: L^{s}(\lambda) \rightarrow L^{s / m}(\lambda)$ be a rightto-left $G$-invariant, positive $m$-homogeneous polynomial. Then $P$ can be defined as a polynomial from $L_{r}(\lambda)$ to $L_{r / m}(\lambda)$.

Using Corollary 4.2 and taking into account that the mixed space

$$
L_{s_{1}}\left(\lambda_{1}\right)\left[L_{s_{2}}\left[\lambda_{2}\right]\right.
$$

is $r$-convex for $r \leq \min \left\{s_{1}, s_{2}\right\}$, the following version of the previous result can be obtained. Clearly, $\left(L_{s_{1}}\left(\lambda_{1}\right)\left[L_{s_{2}}\left(\lambda_{2}\right)\right]\right)^{r}=L_{s_{1} / r}\left(\lambda_{1}\right)\left[L_{s_{2} / r}\left(\lambda_{2}\right)\right]$. For the proof, notice that in Theorem 5.6, matching with respect to the left action of the group can be changed by matching with respect to the right action (by Corollary 4.2), whenever the operator is right-to-right $G$-invariant instead of right-to-left $G$-invariant.

Corollary 5.8. Let $G_{1}$ and $G_{2}$ be amenable locally compact groups with left Haar measures $\lambda_{1}$ and $\lambda_{2}$, respectively. Let $1 \leq r \leq \min \left\{s_{1}, s_{2}\right\}<\infty, m \in \mathbb{N}$ and $1 \leq r / m$. Let $P: L_{s_{1}}\left(\lambda_{1}\right)\left[L_{s_{2}}\left(\lambda_{2}\right)\right] \rightarrow L_{s_{1} / m}\left(\lambda_{1}\right)\left[L_{s_{2} / m}\left(\lambda_{2}\right)\right]$ be a right-to-right $G_{1} \times G_{2}$-invariant, positive m-homogeneous polynomial.

Then $P$ extends to a positive $m$-homogeneous polynomial from $L_{r}\left(\lambda_{1} \times \lambda_{2}\right)$ to $L_{r / m}\left(\lambda_{1} \times \lambda_{2}\right)$.

5.3. Multilinear mappings. Motivated by the previous ideas we study in what follows translation invariant multilinear operators. To do this we need to introduce some notation. 
Let $G$ be a locally compact group with left Haar measure $\lambda$ which acts on $\left(\Omega_{k}, \Sigma_{k}, \mu_{k}\right), 1 \leq k \leq m$, and $(\Upsilon, \Sigma, \nu)$. Assume that $X_{1}, \ldots, X_{m}$ are weak-Ginvariant quasi-Banach function lattices over $\left(\Omega_{k}, \mu_{k}\right)$, respectively, and $Y$ a weak$G$-invariant quasi-Banach function space over $(\Upsilon, \nu)$. Moreover, let $E_{1}, \ldots, E_{m}$ and $F$ be quasi-Banach spaces. A multilinear map $T: X_{1}\left(E_{1}\right) \times \cdots \times X_{m}\left(E_{m}\right) \rightarrow Y(F)$ is said to be translation invariant whenever

$$
\left\|\tau_{x}\left(T\left(f_{1}, \ldots, f_{m}\right)\right)(\cdot)\right\|_{F}=\left\|T\left(\tau_{x} f_{1}, \ldots, \tau_{x} f_{m}\right)(\cdot)\right\|_{F}
$$

for all $\left(f_{1}, \ldots, f_{m}\right) \in X_{1} \times \cdots \times X_{m}$ and all $x \in G$. In the case that the action considered is the group on itself, we use the expression $G$-invariant as in the previous sections, taking into account the right/left actions in the domain an the range spaces.

We need the following technical lemma.

Lemma 5.9. Let $0<r, r_{1}, \ldots, r_{m}<\infty$, and let

$$
\Phi: X_{1}\left(E_{1}\right) \times \cdots \times X_{m}\left(E_{m}\right) \rightarrow Y(F)
$$

be a G-invariant map for which there exist nonnegative weights $w \in L^{0}(\nu)$ and $w_{k} \in L^{0}\left(\mu_{k}\right), 1 \leq k \leq m$, such that for all $f_{k} \in X_{k}\left(E_{k}\right), 1 \leq k \leq m$,

$$
\int_{\Upsilon}\left\|\Phi\left(f_{1}, \ldots, f_{n}\right)(\cdot)\right\|_{F}^{r} w d \nu \leq \sum_{k=1}^{m} \int_{\Omega}\left\|f_{k}(\cdot)\right\|_{E_{k}}^{r_{k}} w_{k} d \mu_{k} .
$$

Then for all nonnegative $g_{k} \in L^{0}(G)$ and all $f_{k} \in X_{k}\left(E_{k}\right), 1 \leq k \leq m$, we have

$$
\int_{\Upsilon}\left\|\Phi\left(f_{1}, \ldots, f_{m}\right)(\cdot)\right\|_{F}^{r}\left(\sum_{k=1}^{m} g_{k} \circledast w\right) d \nu \leq \sum_{i, k=1}^{m} \int_{\Omega}\left\|f_{k}(\cdot)\right\|_{E_{k}}^{r_{k}}\left(g_{i} \circledast w_{k}\right) d \mu_{k} .
$$

Proof. We apply Fubini's Theorem to deduce that

$$
\begin{aligned}
& \int_{\Upsilon}\left\|\Phi\left(f_{1}, \ldots, f_{m}\right)(\cdot)\right\|_{F}^{r}\left(\sum_{k=1}^{m} g_{k} \circledast w\right) d \nu \\
& =\sum_{k=1}^{m} \int_{G} g_{k}(x)\left(\int_{\Upsilon}\left(\left\|\Phi\left(f_{1}, \ldots, f_{m}\right)(x \diamond \omega)\right\|_{F}^{r}\right) w(\omega) d \nu(\omega)\right) d \lambda(x) \\
& =\sum_{k=1}^{m} \int_{G} g_{k}(x)\left(\int_{\Upsilon}\left(\left\|\tau_{x}\left(\Phi\left(f_{1}, \ldots, f_{m}\right)\right)(\omega)\right\|_{F}^{r}\right) w(\omega) d \nu(\omega)\right) d \lambda(x) \\
& =\sum_{k=1}^{m} \int_{G} g_{k}(x)\left(\int_{\Upsilon}\left(\left\|\Phi\left(\tau_{x} f_{1}, \ldots, \tau_{x} f_{m}\right)(\omega)\right\|_{F}^{r}\right) w(\omega) d \nu(\omega)\right) d \lambda(x) \\
& \leq \sum_{k=1}^{m} \int_{G} g_{k}(x)\left(\sum_{i=1}^{m} \int_{\Omega}\left\|f_{i}(\omega)\right\|_{E_{i}}^{r_{k}} w_{i}(\omega) d \mu_{i}(\omega)\right) d \lambda(x) \\
& =\sum_{i, k=1}^{m} \int_{G}\left\|f_{i}(\cdot)\right\|_{E}^{r}\left(g_{k} \circledast w_{i}\right) d \mu_{i},
\end{aligned}
$$

which proves the result. 
We need to modify the definition of matching in the multilinear setting. We say that a finite family $\left\{X_{1}, \ldots, X_{m}\right\}$ of Banach function lattices over $(G, \lambda)$ matches a Banach function lattice $Y$ over $(G, \lambda)$ if there exists a constant $c>0$ such that for every compact set $K \subset G$ there are nonnegative functions $g_{k} \in X_{k}$ and $h \in S_{Y^{\prime}}$ for which $\left\|g_{k}\right\|_{X_{k}} \leq c$ and $g_{k} \circledast h(x)=1$ for all $x \in K$ and for each $1 \leq k \leq m$. We note that this notion depends on the action that is considered. Note that whenever $X_{1}=\ldots=X_{m}$, then the results from Section 4 give various examples.

Theorem 5.10. Let $G$ be a locally compact group with left Haar measure $\lambda$. Let $X_{k}, 1 \leq k \leq m$ be left $G$-invariant order continuous Banach function lattices on $G$, and $Y$ a left $G$-invariant Banach function lattice on $G$. Let $1 \leq r \leq r_{k}<\infty$ such that $1 / r=1 / r_{1}+\cdots+1 / r_{m}$. Suppose that the family $\left\{X_{1}^{r_{1}}, \ldots, X_{1}^{r_{m}}\right\}$ matches $Y^{r}$ (with respect to the left action of $G$ ) $, X_{1}, \ldots, x_{m}$ are $r_{i}$-convex and $Y$ is $r$-convex. If $E_{1}, \ldots, E_{m}$ and $F$ are quasi-Banach spaces and $T: X_{1}\left(E_{1}\right) \times \cdots \times X_{m}\left(E_{m}\right) \rightarrow Y$ is a right-to-left $G$-invariant multilinear map, then $T$ extends to a multilinear map

$$
T: L^{r_{1}}\left(\lambda, E_{1}\right) \times \cdots \times L^{r_{m}}\left(\lambda, E_{m}\right) \rightarrow L^{r}(\lambda, F)
$$

provided that there exists a constant $C>0$ such that for every choice of finitely many sequences $\left\{x_{j}^{(i)}\right\}_{j=1}^{n}$ in $X_{i}, 1 \leq i \leq m$, we have

$$
\begin{aligned}
& \left\|\left(\sum_{j=1}^{n}\left\|T\left(x_{j}^{(1)}, \ldots, x_{j}^{(m)}\right)(\cdot)\right\|_{F}^{r}\right)^{1 / r}\right\|_{Y} \\
& \leq C\left\|\left(\sum_{j=1}^{n}\left\|x_{j}^{(1)}(\cdot)\right\|_{E_{1}}^{r_{1}}\right)^{1 / r_{1}}\right\|_{X_{1}} \ldots\left\|\left(\sum_{j=1}^{n}\left\|x_{j}^{(m)}(\cdot)\right\|_{E_{m}}^{r_{m}}\right)^{1 / r_{m}}\right\|_{X_{m}} .
\end{aligned}
$$

Proof. As in previous results, we can assume without loss of generality that the $s$-convexity constants of the spaces involved equal one. Fix a compact subset $K$ of $G$, and choose (according to the definition) a constant $c>0$ (independent of $K$ ) and nonnegative functions $h \in S_{\left(Y^{r}\right)^{\prime}}$ and $g_{i} \in X_{k}^{r_{i}}$ with $\left\|g_{i}\right\|_{X_{i}^{r_{i}}} \leq c$ such that $g_{i} \circledast h=1$ on $K$. Notice that $\left(\left(Y^{r}\right)^{\prime}\right)^{1 / r}=M\left(Y, L^{r}\right)$ up to equivalence of norms (see again the proof of Proposition 5.1). Consider the multilinear map

$$
M_{h^{1 / r}} \circ T: X_{1}\left(E_{1}\right) \times \cdots \times X_{m}\left(E_{m}\right) \rightarrow L_{r}(F) .
$$

A minor modification of the proof of Theorem 3.5 in [24] (in fact this is a result for scalar-valued functions which easily extends to the vector-valued case) and our hypothesis on the vector-valued inequality for $T$ give that there exists a constant $M$ and a nonnegative weight $w_{i}$ in the unit ball of $\left(X^{r_{i}}\right)^{\prime}$ for each $1 \leq i \leq m$ such that

$$
\int_{G}\left\|T\left(x_{1}, \ldots, x_{m}\right)(\cdot)\right\|_{F}^{r} h d \lambda \leq M \sum_{i=1}^{m} \frac{r}{r_{i}} \int_{G}\left\|x_{i}(\cdot)\right\|_{E_{i}}^{r_{i}} w_{i} d \lambda
$$


Now we apply Lemma 5.9 and Proposition 3.1 (taking into account the correct election of either the right or the left action in each step) and obtain

$$
\begin{aligned}
\int_{K}\left\|T\left(x_{1}, \ldots, x_{m}\right)(\cdot)\right\|_{F}^{r} d \lambda & =\int_{K}\left\|T\left(x_{1}, \ldots, x_{m}\right)(\cdot)\right\|_{F}^{r}\left(\sum_{k=1}^{m} \frac{r}{r_{k}} g_{k} \circledast h\right) d \lambda \\
& \leq M \sum_{k, i=1}^{m} \frac{r}{r_{k}} \int_{G}\left\|x_{i}(\cdot)\right\|_{E_{i}}^{r} g_{k} \circledast w_{i} d \lambda \\
& \leq c M \sum_{k, i=1}^{m} \frac{r}{r_{k}} \int_{G}\left\|x_{i}(\cdot)\right\|_{E_{i}}^{r_{i}} d \lambda .
\end{aligned}
$$

In particular, if $\left\|x_{i}\right\|_{L_{r_{i}}\left(E_{i}\right)} \leq 1$ for each $1 \leq i \leq m$, then we get that

$$
\left(\int_{G}\left\|T\left(x_{1}, \ldots, x_{m}\right)(\cdot)\right\|_{F}^{r} d \lambda\right)^{1 / r} \leq c M m
$$

and this completes the proof.

Let us give an application for positive multilinear maps. At first note that for any positive multilinear map $T: X_{1} \times \cdots \times X_{m} \rightarrow Y$ in Banach function lattices the following vector-valued inequality holds true (see [11, Theorem 6.2]):

$$
\begin{aligned}
& \left\|\left(\sum_{j=1}^{n}\left|T\left(x_{j}^{(1)}, \ldots, x_{j}^{(m)}\right)\right|^{r}\right)^{1 / r}\right\|_{Y} \\
& \leq\|T\|\left\|\left(\sum_{j=1}^{n}\left|x_{j}^{(1)}\right|^{r_{1}}\right)^{1 / r_{1}}\right\|_{X_{1}} \ldots\left\|\left(\sum_{j=1}^{n}\left|x_{j}^{(m)}\right|^{r_{m}}\right)^{1 / r_{m}}\right\|_{X_{m}} .
\end{aligned}
$$

Applying this to the preceding theorem in the scalar case (i.e., $E_{i}=F=\mathbb{R}, 1 \leq$ $i \leq m$ ) we obtain the following consequence.

Corollary 5.11. Suppose that the Banach function lattices $X_{1}, \ldots, X_{m}$ and $Y$ satisfy the assumptions of Theorem 5.10. Then every right-to-left $G$-invariant, positive multilinear map $T: X_{1} \times \cdots \times X_{m} \rightarrow Y$ extends to a positive multilinear map

$$
T: L^{r_{1}}(\lambda) \times \cdots \times L^{r_{m}}(\lambda) \rightarrow L^{r}(\lambda)
$$

\section{Applications to operators}

Finally, we collect a few applications to translation invariant linear operators in vector-valued quasi-Banach function lattices which seem to be of independent interest.

6.1. Linear operators in Köthe-Bochner spaces. We intend to apply Theorem 5.3 , and for that we isolate pairs of spaces $(\mathcal{X}, \mathcal{Y})$ such each operator from $\mathcal{X}$ to $\mathcal{Y}$ satisfies the appropriate vector-valued norm inequality. 
Let us introduce some additional notation: Given a 1-homogeneous mapping $\Phi: X(\mu, E) \rightarrow Y(\nu, F)$ between vector-valued quasi-Banach function spaces and $0<r<\infty$ we denote by

$$
\mathbf{m}_{r}(\Phi)=\mathbf{m}_{r}(\Phi: X(E) \rightarrow Y(F))
$$

the best constant $M>0$ such for all possible choices of sequences $\left\{f_{k}\right\}_{k=1}^{n}$ in $X(E)$

$$
\left\|\left(\sum_{k=1}^{n}\left\|\Phi f_{k}(\cdot)\right\|_{F}^{r}\right)^{1 / r}\right\|_{Y} \leq M\left\|\left(\sum_{k=1}^{n}\left\|f_{k}(\cdot)\right\|_{E}^{r}\right)^{1 / r}\right\|_{X}
$$

The central tools for proving that $\mathbf{m}_{r}(\Phi)<\infty$ are based on local Banach spaces theory. In particular we use the notions of (Rademacher) type and cotype, as well as stable type. For details we refer to the monographs [9, 14].

Lemma 6.1. Let $0<p, q, r<\infty$, and let $E$ and $F$ be Banach spaces. For any linear operator $T: L_{q}(\mu, E) \rightarrow L_{p}(\nu, F)$ we have $\mathbf{m}_{r}(T)<\infty$ whenever one of the following conditions is satisfied:

(i) $1 \leq p, q<\infty, r=2$ and $E$ has type 2 and $F$ has cotype 2 .

(ii) $0<p<\infty, 0<q<r<2$ and $E$ has stable type $r$.

(iii) $1<q<\infty, 2<r<p<\infty$, $F^{*}$ has stable type $r^{\prime}$ and moreover $T^{*}\left(L_{p^{\prime}}\left(\nu, F^{*}\right)\right) \subset L_{q^{\prime}}\left(\mu, E^{*}\right)$.

Proof. We sketch the proof, and start with $(i)$. The definition of type 2 and cotype 2, combined with Kahane's inequality on the equivalence of Banach space-valued Rademacher averages (see, e.g., [14, p.211]), shows that the statement holds in the case $1 \leq p \leq 2 \leq q<\infty$. For the general case define $p_{1}=\min \{2, p\}, q_{1}=\max \{2, q\}$ as well as $\frac{1}{r_{1}}=\frac{1}{q}-\frac{1}{q_{1}}, \frac{1}{r_{2}}=\frac{1}{p_{1}}-\frac{1}{p}$.

Recall that $r=2$. Given $f_{1}, \ldots, f_{n} \in L_{q}(\mu, E)$ choose some nonnegative norm one functions $g_{1} \in L_{r_{1}}(\mu)$ and $g_{2} \in L_{r_{2}}(\nu)$ such that

$$
\left(\int\left(\sum_{k=1}^{n}\left\|f_{k}(\cdot)\right\|_{E}^{2}\right)^{q_{1} / 2} g_{1}^{-q_{1}} d \mu\right)^{1 / q_{1}}=\left(\int\left(\sum_{k=1}^{n}\left\|f_{k}(\cdot)\right\|_{E}^{2}\right)^{q / 2} d \mu\right)^{1 / q}
$$

and

$$
\left(\int\left(\sum_{k=1}^{n}\left\|T f_{k}(\cdot)\right\|_{F}^{2}\right)^{p_{1} / 2} g_{2}^{p_{1}} d \nu\right)^{1 / p_{1}}=\left(\int\left(\sum_{k=1}^{n}\left\|T f_{k}(\cdot)\right\|_{F}^{2}\right)^{p / 2} d \nu\right)^{1 / p} .
$$


Then the desired inequality follows from the previous case:

$$
\begin{aligned}
& \left(\int\left(\sum_{k=1}^{n}\left\|T f_{k}(\cdot)\right\|_{F}^{2}\right)^{p / 2} d \nu\right)^{1 / p} \\
& =\left(\int\left(\sum_{k=1}^{n}\left\|\left(M_{g_{2}} \circ T \circ M_{g_{1}}\right)\left(g_{1}^{-1} f_{k}\right)(\cdot)\right\|_{F}^{2}\right)^{p_{1} / 2} d \nu\right)^{1 / p_{1}} \\
& \leq\left(\int\left(\sum_{k=1}^{n}\left\|\left(g_{1}^{-1} f_{k}\right)(\cdot)\right\|_{E}^{2}\right)^{q_{1} / 2} d \mu\right)^{1 / q_{1}}=\left(\int\left(\sum_{k=1}^{n}\left\|f_{k}(\cdot)\right\|_{E}^{2}\right)^{q / 2} d \mu\right)^{1 / q} .
\end{aligned}
$$

The proof of ( $i$ i ) follows similar lines: check first of all the case $0<p<q<r<2$ using the definition of stable type (and a variant of Kahane's inequality for stable measures), and then in the second step copy the previous trick. The last statement follows by some standard duality arguments.

Combining Lemma 6.1 with Theorem 5.3 we obtain the following result which is of different nature than Corollary 5.8.

Theorem 6.2. Let $G$ be an amenable locally compact group with (left) Haar measure $\lambda$, and let $T: L_{q}\left(\lambda, L_{u}\left(\nu_{1}\right)\right) \rightarrow L_{q}\left(\lambda, L_{v}\left(\nu_{2}\right)\right)$ be a bounded right-to-left $G$ invariant linear operator.

Then $T$ extends to an operator from $L_{r}\left(\lambda, L_{u}\left(\nu_{1}\right)\right)$ into $L_{r}\left(\lambda, L_{v}\left(\nu_{2}\right)\right)$ whenever one of the following conditions is satisfied:

(i) $r=2,1 \leq v \leq 2 \leq u \leq \infty$ and $1<q<\infty$

(ii) $r<2,1 \leq q<r<u<\infty$

(iii) $r>2,1<v<r<q<\infty$ and $1<u<\infty$

Recall that this result can be improved when the group $G$ is compact by using Corollary 5.5.

6.2. Homogeneous operators in Lorentz and Orlicz spaces. This subsection provides application in the setting of Lorentz and Orlicz spaces. We start with the observation that every r.i. space $X$ over a compact group $G$ is $G$-invariant. In fact, consider the left action of $G$ on itself, then for all $f \in X$ and all $t \geq 0$ we have

$$
\mu_{f}(t)=\lambda(\{y \in G:|f(y)|>t\}), \quad \mu_{\tau_{x} f}(t)=\lambda(\{y \in G:|f(x y)|>t\}) .
$$

Since $\lambda$ is left- $G$-invariant,

$$
\begin{aligned}
\mu_{\tau_{x} f}(t) & =\lambda(\{y \in G:|f(x y)|>t\})=\lambda\left(\left\{x^{-1} z: z \in G,|f(z)|>t\right\}\right) \\
& =\lambda\left(x\left\{x^{-1} z: z \in G,|f(z)|>t\right\}\right)=\mu_{f}(t),
\end{aligned}
$$

and this clearly yields that $X$ is left $G$-invariant. Similar arguments give that $X$ is right $G$-invariant whenever the right action is considered. In particular, Lorentz and Orlicz spaces are $G$-invariant. 
Proposition 6.3. Let $G$ be $n$ unimodular amenable locally compact group with Haar measure $\lambda$, and let $E, F$ be quasi-Banach spaces. For $0<r<p<\infty$, $0<r \leq q_{1}<\infty$ and $0<r \leq q_{2}<\infty$, consider the Lorentz spaces $L_{p, q_{1}}(\lambda)$ and $L_{p, q_{2}}(\lambda)$. Then every homogeneous and right-to-left $G$-invariant operator

$$
\Phi: L_{p, q_{1}}(\lambda, E) \rightarrow L_{p, q_{2}}(\lambda, F)
$$

extends to a homogeneous bounded operator from $L_{r}(\lambda, E)$ to $L_{r}(\lambda, F)$ provided that there is some $K>0$ such that for all choices of functions $f_{1}, \ldots, f_{n} \in L_{p, q_{1}}(\mu, E)$

$$
\left\|\left(\sum_{k=1}^{n}\left\|\Phi f_{k}(\cdot)\right\|_{F}^{r}\right)^{1 / r}\right\|_{L_{p, q_{2}}} \leq K\left\|\left(\sum_{k=1}^{n}\left\|f_{k}(\cdot)\right\|_{E}^{r}\right)^{1 / r}\right\|_{L_{p, q_{1}}}
$$

Proof. We start the proof recalling results about concavity and convexity of Lorentz spaces (see [7]):

(1) If $0<u \leq v \leq \infty$, then $L_{v, u}$ is $u$-convex and $s$-concave for $v<s$.

(2) If $0<v<u \leq \infty$, then $L_{v, u}$ is $u$-concave and $s$-convex for $s<v$.

The $r$-th powers of the $r$-convex spaces $L_{p, q_{1}}(\lambda)$ and $L_{p, q_{2}}(\lambda)$ are $L_{p, q_{1}}(\lambda)^{r}=$ $L_{p / r, q_{1} / r}(\lambda)$ and $L_{p, q_{2}}(\lambda)^{r}=L_{p / r, q_{2} / r}(\lambda)$, respectively. Both of them are Banach function spaces and $L_{p / r, q_{1} / r}(\lambda)$ matches with $L_{p / r, q_{2} / r}(\lambda)$, by Corollary 4.4. Since both spaces are left and right $G$-invariant, Theorem 5.3 completes the proof.

We conclude with an application for Orlicz spaces. At first we remark that convexity and concavity of Orlicz spaces are well characterized; for this we refer to [20] and the references therein.

Proposition 6.4. Let $G$ be an unimodular amenable locally compact group with Haar measure $\lambda$ and let $E, F$ be quasi-Banach spaces. For $0<r<\infty$ let $\varphi$ be a Young function, and suppose that $t \mapsto \varphi\left(t^{1 / r}\right)$ is equivalent to a convex function. Assume that the Orlicz space $L_{\varphi}(\lambda)$ is order continuous. Then the homogeneous and right-to-left $G$-invariant operator

$$
\Phi: L_{\varphi}(\lambda, E) \rightarrow L_{\varphi}(\lambda, F)
$$

extends to a bounded and homogeneous operator from $L_{r}(\lambda, E)$ to $L_{r}(\lambda, F)$ provided that there is some $K>0$ such that for all choices of functions $f_{1}, \ldots, f_{n} \in L_{\varphi}(\lambda, E)$

$$
\left\|\left(\sum_{k=1}^{n}\left\|\Phi f_{k}(\cdot)\right\|_{F}^{r}\right)^{1 / r}\right\|_{L_{\varphi}} \leq K\left\|\left(\sum_{k=1}^{n}\left\|f_{k}(\cdot)\right\|_{E}^{r}\right)^{1 / r}\right\|_{L_{\varphi}} .
$$

Proof. This a direct consequence of the fact that the space $L_{\varphi}(\lambda)$ matches with itself (Lemma 4.3), and that it is $r$-convex whenever $t \mapsto \varphi\left(t^{1 / r}\right)$ is equivalent to a convex function. Then we finish the proof by using the same arguments of the proof of Proposition 6.3 for Lorentz spaces. 
6.3. Pietsch measures. We finish with an application to translation invariant summing operators on $C(G)$-spaces over compact abelian groups. This class is of special interest in harmonic analysis, in particular for some problems related to Hardy $H_{p}$-spaces (see, e.g., the paper by Kwapień and Pełczyński [22]).

Let $F$ be a Banach space and $G$ a compact group. In this context, a linear operator $T: C(G) \rightarrow F$ is said to be translation invariant if $\left(T \circ \tau_{x}\right) f=T f$ for all $x \in G$ and $f \in C(G)$. In what follows we show that our technique improves a classical result on translation invariant summing operators due to Gordon [16] (see also the paper by Pełczyński [31]) which states the following: Let $T: C(G) \rightarrow F$ be a translation invariant, $r$-summing operator. Then the Haar measure $\lambda$ is a Pietsch measure for $T$, i.e., for all $f \in C(G)$

$$
\|T f\|_{F} \leq \pi_{p}(T)\left(\int_{G}|f|^{p} d \lambda\right)^{1 / p}
$$

For the theory of summing operators we refer to the monograph [14], and to [2] and $[14, p .56]$ for extensions of the preceding result in the setting of multilinear summing mappings on closed $G$-invariant subspaces of $C(G)$.

We need a variant of Proposition 5.2 which may be considered as its non-order continuous counterpart, and for the sake of completeness we include a short proof.

Lemma 6.5. Let $G$ be a compact group with Haar measure $\lambda$, and let $Y$ be a weak left-G-invariant quasi-Banach function lattice over $G$. Let $E$ and $F$ be Banach spaces, and let $\Phi: C(G, E) \rightarrow Y(\lambda, F)$ be a left-to-left $G$-invariant map such that there exist $\varphi \in Y^{*}, \psi \in C(G)^{*}$ and a constant $K>0$ such that for all $f \in C(G, E)$

$$
\varphi\left(\|(\Phi f)(\cdot)\|_{F}\right) \leq K \psi\left(\|f(\cdot)\|_{E}\right) .
$$

Then for all nonnegative $g \in C(G)$ and all $f \in C(G, E)$ we have

$$
(g \star \varphi)\left(\|(\Phi f)(\cdot)\|_{F}\right) \leq K(g \star \psi)\left(\|f(\cdot)\|_{E}\right) .
$$

Proof. Fix $g, \varphi$, and $\psi$ as in the statement. From Lemma 3.4 we know that $g \star \psi \in$ $C(G)^{*}$. Applying Fubini's theorem we get that

$$
\begin{aligned}
& (g \star \varphi)\left(\|(\Phi f)(\cdot)\|_{F}\right)=\int_{G} g(x) \varphi\left(\left\|\tau_{x}(\Phi f)(\cdot)\right\|_{F}\right) d \lambda(x) \\
& =\int_{G} g(x) \varphi\left(\left\|\left(\tau_{x} \circ \Phi\right)(f)(\cdot)\right\|_{F}\right) d \lambda(x) \\
& =\int_{G} g(x) \varphi\left(\left\|\Phi\left(\tau_{x} f\right)(\cdot)\right\|_{F}\right) d \lambda(x) \\
& =\int_{G} g(x) \varphi\left(\|\Phi(f(x \cdot))(\cdot)\|_{F}\right) d \lambda(x) \\
& \leq K \int_{G} g(x) \psi\left(\|f(x \cdot)\|_{E}\right) d \lambda(x)=K(g \star \psi)\left(\|f(\cdot)\|_{E}\right),
\end{aligned}
$$

as required. 
Remark 6.6. By Lemma 3.3 we know that if $Y$ in the preceding proposition is also order continuous, then the functional $\varphi$ can be represented by a norm one functional $k \in Y^{\prime}$. Thus $g \star \varphi$ can be identified with $g \circledast k$.

Using the preceding lemma the proof of the next result is similar to the proof of Theorem 5.4 and so we skip it.

Theorem 6.7. Let $G$ be a compact group with (left) Haar measure $\lambda$, and let $Y$ be an order continuous, weak left-G-invariant, $r$-convex Banach function lattice over $G$. Suppose that $E$ and $F$ are quasi-Banach spaces and $T: C(G, E) \rightarrow Y(\lambda, F)$ is a left-to-left $G$-invariant $m$-homogeneous operator $T: C(G, E) \rightarrow Y(\lambda, F)$ satisfying the inequality

$$
\left\|\left(\sum_{k=1}^{n}\left\|T f_{k}\right\|_{F}^{r / m}\right)^{m / r}\right\|_{Y} \leq K\left\|\left(\sum_{k=1}^{n}\left\|f_{k}(\cdot)\right\|_{E}^{r}\right)^{1 / r}\right\|_{C(G)}^{m} .
$$

Then $T$ extends to a bounded operator from $L_{r}(\lambda, E)$ into $L_{r / m}(\lambda, F)$.

As an application we recover the recent result of $[2,4.2]$. In the case when $m=1$ this yields the result of Gordon-Pełczyński.

Corollary 6.8. Given a Banach space $F$ and a compact group $G$, let $P: C(G) \rightarrow F$ be a translation invariant, $r$-dominated and $m$-homogeneous polynomial, i.e., there is $K>0$ such that for every choice of finitely many $f_{1}, \ldots, f_{n} \in C(G)$ we have

$$
\left(\sum_{k=1}^{n}\left\|P f_{k}(\cdot)\right\|_{F}^{r / m}\right)^{m / r} \leq K \sup _{t \in G}\left(\sum_{k=1}^{n}\left|f_{k}(t)\right|^{r}\right)^{m / r} .
$$

Then the Haar measure $\lambda$ is a Pietsch measure for P, i.e.,

$$
\|P f\|_{F} \leq K\left(\int_{G}|f|^{r} d \lambda\right)^{m / r}, \quad f \in C(G) .
$$

Proof. Consider the $m$-homogeneous operator $\widehat{P}: C(G) \rightarrow L_{r}(\lambda, F)$ given by

$$
\widehat{P}=\chi_{G} \otimes P f, \quad f \in C(G) .
$$

Since $P$ is translation invariant, we deduce that $\widehat{P}$ is left-to-left $G$-invariant. Moreover, since $T$ is $r$-dominated, we get that for every choice of finitely many functions $f_{1}, \ldots, f_{n} \in C(G)$

$$
\begin{aligned}
\left\|\left(\sum_{k=1}^{n}\left\|\widehat{P} f_{k}(\cdot)\right\|_{F}^{r / m}\right)^{m / r}\right\|_{L_{r / m}} & =\left(\sum_{k=1}^{n}\left\|P f_{k}(\cdot)\right\|_{F}^{r / m}\right)^{m / r} \\
& \leq K\left\|\left(\sum_{k=1}^{n}\left|f_{k}\right|^{r}\right)^{1 / r}\right\|_{C(G)}^{m} .
\end{aligned}
$$

Then Theorem 6.7 gives that $\widehat{P}$ in fact extends to an operator from $L_{r}(\lambda)$ to $L_{r / m}(\lambda, F)$. By the definition of $\widehat{P}$ we obtain that $P$ is well-defined and bounded 
from $L_{r}(\lambda)$ to $F$, and so

$$
\|P f\|_{F} \leq K\left(\int_{G}|f|^{r} d \lambda\right)^{m / r}, \quad f \in C(G),
$$

which completes the proof.

Acknowledgement. We thank the referee for careful reading of the paper and useful remarks.

\section{REFERENCES}

[1] C. Bennett and R. Sharpley, Interpolation of Operators, Pure and Applied Mathematics, Vol. 119, Academic Press, Boston, 1988.

[2] G. Bothelho, D. Pellegrino, P. Rueda, J. Santos and J. B. Seoane-Sepúlveda, When is the Haar measure a Pietsch measure for nonlinear mappings?, Studia Math. 213 (2012), no. 3, 275-287.

[3] L. Colzani, Translation invariant operators on Lorentz spaces, Ann. Scuola Norm. Sup. Pisa Cl. Sci. (4) 14 (1987), no. 2, 257-276 (1988).

[4] L. Colzani and P. Sjögren, Translation-invariant operators on Lorentz spaces $L(1, q)$ with $0<q<1$, Studia Math. 132 (1999), no. 2, 101-124.

[5] M. Cowling, Some applications of Grothendieck's theory of topological tensor products in harmonic analysis, Math. Ann. 232 (1978), no. 3, 273-285.

[6] M. G. Cowling and John J.F. Fournier, Inclusions and noninclusion of spaces of convolution operators, Trans. Amer. Math. Soc. 221 (1976), no. 1, 59-95.

[7] J. Creekmore, Type and copype of Lorentz $L_{p q}$ spaces, Indag. Math. 43 (1981), 145-152.

[8] A. Defant, Variants of the Maurey-Rosenthal theorem for quasi Köthe function spaces, Positivity 5 (2001), no. 2, 153-175.

[9] A. Defant and K. Floret, Tensor norms and operator ideals, volume 176 of North-Holland Mathematics Studies. North-Holland Publishing Co., Amsterdam, 1993.

[10] A. Defant anf M. Junge, Best constants and asymptotics of Marcinkiewicz-Zygmund inequalities, Studia Math. 125 (1997), no. 3, 271-287.

[11] A. Defant and M Mastyło, Interpolation of Fremlin tensor products and Schur factorization of matrices, J. Funct. Anal. 262 (2012), 3981-3999.

[12] A. Defant and M. Mastyło, Factorization and extension of positive homogeneous polynomials, Studia Math. 221 (2014), no. 1, 87-100.

[13] A. Defant and E. A. Sánchez-Pérez, Maurey-Rosenthal factorization of positive operators and convexity, J. Math. Anal. Appl. 297 (2004), no. 2, 771-790.

[14] J. Diestel, H. Jarchow and A. Tonge, Absolutely Summing Operators, Cambridge Studies in Advanced Math. no. 43. Cambridge 1995.

[15] J. García-Cuerva, and J.L. Rubio de Francia, Weighted Norm Inequalities and Related Topics, North-Holland Mathematics Studies 116. Amsterdam 1985.

[16] Y. Gordon, On p-summing constants of Banach spaces, Israel Journ. Math. 7 (1969), 151163.

[17] L. Grafakos, Classical and modern Fourier analysis, Pearson Education, Inc., Upper Saddle River, NJ, 2004. 
[18] C. Herz, The theory of p-spaces with an application to convolution operators, Trans. Amer. Math. Soc. 154 (1971), 69-82.

[19] C. Herz and and N. Rivière, Estimates for translation-invariant operators on spaces with mixed norms, Studia Math. 44 (1972), 511-515.

[20] A. Kamińska, Indices, convexity and concavity in Musielak-Orlicz spaces, Funct. Approx. Comment. Math. 26 (1998), 67-84.

[21] L. V. Kantorovich and G.P. Akilov, Functional Analysis, Second edition, Pergamon Press, Oxford-Elmsford, N.Y., 1982.

[22] S. Kwapień and A. Pełczyński, Remarks on absolutely summing translation invariant operators from the disc algebra and its dual into a Hilbert space, Mich. Math. Journ. 25 (1978) 173-181.

[23] R. Larsen, An introduction to the theory of multipliers, Springer, Die Grundlagen der Math. Wissenschaften 175 (1975).

[24] M. Mastyło and E. A. Sánchez Pérez, Domination and factorization of multilinear operators, J. Convex Anal. 20 (2013), no. 4, 999-1012.

[25] B. Maurey, Théorèmes de factorisation pour les opérateurs linéairesà valeurs dans les spaces $L_{p}$, Asterisque 11 (1974).

[26] D. M. Oberlin, Translation-invariant operators on $L_{p}(G), 0<p<1$, Michigan Math. J. 23 (1976), no. 2, 119-122.

[27] D. M. Oberlin, Translation-invariant operators on $L_{p}(G), 0<p<1$. II, Canad. J. Math. 29 (1977), no. 3, 626-630.

[28] D. M. Oberlin, Translation-invariant operators of weak type, Pacific J. Math. 85 (1979), no. 1, $155-164$.

[29] S. Okada, W. J. Ricker and E. A. Sánchez Pérez, Optimal domain and integral extension of operators: acting in function spaces. Operator Theory: Advances and Applications, 180. Birkhauser Verlag, Basel, 2008.

[30] Open problems at the first seminar Poland-GDR on operator ideals and geometry of Banach spaces Forschungsergebnisse, 1982, N/82/27.

[31] A. Pełczyński, p-integral operators commuting with group representations and examples of quasi $p$-integral operators which are not p-integral, Studia Math. 28 (1969), 63-70.

[32] H. P. Rosenthal, On subspaces of $L^{p}$, Ann. of Math. 97 (1973), 344-373.

[33] A. M. Shteĭnberg, Translation-invariant operators in Lorentz spaces, Funktsional. Anal. i Prilozhen. 20 (1986), no. 2, 92-93 (in Russian); English transl.: unctional Anal. Appl. 20 (1986), 166-168.

[34] P. Sjögren, Translation-invariant operators on weak $L^{1}$, J. Funct. Anal. 89 (1990), no. 2, $410-427$. 
Institut für Mathematik, Carl von Ossietzky Universität, Postfach 2503, D-26111 Oldenburg, Germany

E-mail address: andreas.defant@uni-oldenburg.de

Faculty of Mathematics and Computer Science, Adam Mickiewicz University in Poznań, Umultowska 87, 61-614 Poznań, Poland

E-mail address: mastylo@amu.edu.pl

Instituto Universitario de Matemática Pura y Aplicada, Universitat Politècnica de València, Camino de Vera s/n, 46022 Valencia, Spain

E-mail address: easancpe@mat.upv.es

Fachbereich Mathematik, Universität Stuttgart, Pfaffenwaldring, D-70569 Stuttgart, GERMANY

E-mail address: Ingo.Steinwart@mathematik.uni-stuttgart.de 